\title{
Inverse limits of tentlike maps on trees
}

by

\author{
Stewart Baldwin (Auburn, AL)
}

\begin{abstract}
We investigate generalizations of Ingram's Conjecture involving maps on trees. We show that for a class of tentlike maps on the $k$-star with periodic critical orbit, different maps in the class have distinct inverse limit spaces. We do this by showing that such maps satisfy the conclusion of the Pseudo-isotopy Conjecture, i.e., if $h$ is a homeomorphism of the inverse limit space, then there is an integer $N$ such that $h$ and $\widehat{\sigma}^{N}$ switch composants in the same way, where $\widehat{\sigma}$ is the standard shift map of the inverse limit space.
\end{abstract}

1. Introduction. One topic which has attracted a great deal of attention in recent years is the problem of distinguishing the topology of the inverse limits of different maps. Notable in this regard are the frequent attempts to solve Ingram's Conjecture and the numerous results which this has generated.

InGRAM's ConjeCture: For a parameter $\lambda \in(1,2]$, let $f_{\lambda}$ be the "tent" map on the unit interval defined as follows:

$$
f_{\lambda}(x)= \begin{cases}\lambda x, & 0 \leq x \leq 1 / 2, \\ \lambda(1-x), & 1 / 2 \leq x \leq 1 .\end{cases}
$$

Then it is conjectured that for $\lambda \neq \nu$ both in $(1,2]$, the inverse limit spaces of $f_{\lambda}$ and $f_{\nu}$ are not homeomorphic (see [I]).

Early results included those of Barge and Martin, who showed that in the cases where the critical point was periodic, the inverse limit space had the same number of endpoints as the period, thus showing that different periods led to different inverse limit spaces [BM]. Working in the case where the turning point had finite orbit, Barge and Diamond showed that if $\mathbb{Q}(\lambda)$ and $\mathbb{Q}(\nu)$ were different algebraic extensions of the rational numbers, then $f_{\lambda}$ and $f_{\nu}$ had nonhomeomorphic inverse limits. Using this, they showed that

2010 Mathematics Subject Classification: Primary 54F15, 54F65; Secondary 37B10. Key words and phrases: Ingram's Conjecture, inverse limit. 
the three parameter values giving period 5 critical orbits all had distinct inverse limits $[\mathrm{BaD}]$. Working in the case where the orbit of the turning point was finite (but not necessarily periodic), Bruin showed that if $\log \lambda$ and $\log \nu$ were rationally independent, then $f_{\lambda}$ and $f_{\nu}$ had nonhomeomorphic inverse limits. Kailhofer showed that Ingram's Conjecture was true for all $\lambda$ giving a periodic critical point [K1, K2], the proof of which was simplified considerably by a paper of Block, Jakimovik, Kailhofer, and Keesling [BJKK]. Stimac extended Ingram's Conjecture to the case where the turning point was preperiodic [S1, S2], and this was then further extended by Raines and Stimac to the case where the turning point was nonrecurrent [RS]. The referee has informed me that a proof of Ingram's Conjecture has recently been announced by Marcy Barge, Henk Bruin, and Sonja Štimac [BBS].

The most detailed investigations in inverse limit spaces have been with maps on the interval. In this paper, we examine the case of a periodic critical point for certain maps on trees which come from a natural generalization of the family of tent maps.

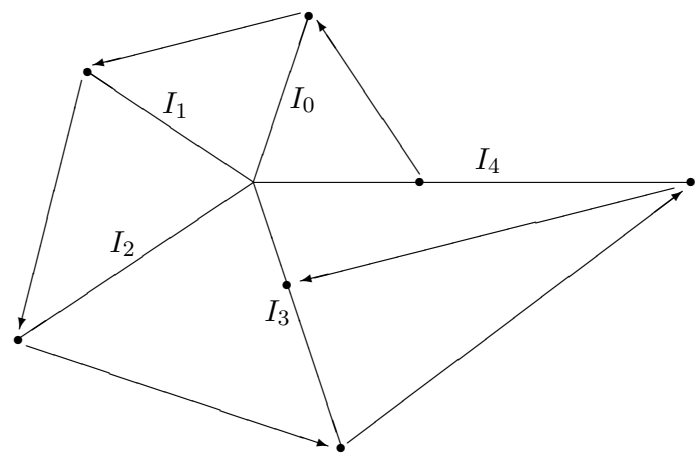

Fig. 1. A function of the family $\phi_{5,3, \lambda}$

Definition 1.1. Define the families $\phi=\phi_{k, j, \lambda}$ of maps on the $k$-star $S_{k}(k \geq 2)$ as follows, $1 \leq j \leq k-1,2<\lambda^{k} \leq 2+\lambda^{j}$. Let $I_{0}, I_{1}, \ldots, I_{k-1}$ be the intervals whose union forms the $k$-star, with center point $c$. Put a taxicab metric on $S_{k}$ by letting $I_{m}$ have length $\lambda^{m+1}$. The center point $c$ is a fixed point of $\phi$. For $0 \leq m \leq k-2$, we let $\phi$ map $I_{m}$ linearly onto $I_{m+1}$ with expansion factor $\lambda$. Let $I_{k-1}=[c, v]$ and let $t$ be the point of $I_{k-1}$ such that $[c, t]$ has length 1 , and let $u$ be the point on $I_{k-1}$ between $t$ and $v$ such that $[t, u]$ has length 1 . (The requirement that $2<\lambda^{k}$ guarantees that $I_{k-1}$ has such points.) We let $\phi(u)=c$, and map each of the intervals $[c, t]$ and $[t, u]$ linearly onto $I_{0}$ with expansion factor $\lambda$. The interval $[u, v]$ is then mapped linearly with expansion factor $\lambda$ into $I_{j}$, however far it reaches (the condition $\lambda^{k} \leq 2+\lambda^{j}$ guaranteeing that it will not reach too far). 
The maps $\phi_{k, j, \lambda}$ are a natural generalization of the tent maps $f_{\lambda}$. In fact, it is easily seen that the maps $\phi_{2,1, \lambda}$ are just rescaled "cores" of the corresponding tent maps $f_{\lambda}$. For $\sqrt{2}<\lambda \leq 2$, the core of the map $f_{\lambda}$ is defined to be $f_{\lambda}$ restricted to the interval $[\lambda(1-\lambda / 2), \lambda / 2]$. The core map is locally eventually onto, and the inverse limit of the core is indecomposable (see below for the definitions). The inverse limit of the full tent map on $[0,1]$ is just the inverse limit of the core with an additional ray which limits on the core.

The maps $\phi_{k, j, \lambda}$ cover more territory than what appears at first glance. As will be shown below, any locally eventually onto continuous function on a star having exactly one turning point which is not the branching point is conjugate to one of the $\phi_{k, j, \lambda}$ 's, with $j$ relatively prime to $k$. The main result of this paper will be to show that the $\phi_{k, j, \lambda}$ 's have distinct inverse limit spaces in the case where the turning point is periodic. The present paper owes much to the recent paper of Block, Jakimovik, Kailhofer, and Keesling [BJKK], and also to Kailhofer's original papers [K1, K2]. However, there are also some major differences between the arguments of those papers and the arguments appearing here, which are heavily based on the composant containing the fixed point of the inverse limit shift map. Unlike the case where $k=2$, this fixed point is topologically identifiable in the inverse limits spaces of the $\phi_{k, j, \lambda}$ for $k \geq 3$, and that leads to some significant differences in the details. (Here, we are talking about the fixed point that comes from the core, and not the fixed point of the limiting ray.)

In their simplification of the argument for the case of a periodic critical orbit, Block, Jakimovik, Kailhofer, and Keesling looked closely at how composants of the inverse limit were mapped by an arbitrary homeomorphism, showing that composants were mapped in the same way as by some iterate of the natural shift map [BJKK]. This led to the following related conjecture.

The Pseudo-isotopy Conjecture. Let $\lambda \in(\sqrt{2}, 2]$, and let $f$ be the core of the slope $\lambda$ tent map on the interval. Let $\sigma: \widehat{X} \rightarrow \widehat{X}$ be the shift map of the inverse limit space of $f$, and let $h: \widehat{X} \rightarrow \widehat{X}$ be another homeomorphism of $\widehat{X}$. Then there is an integer $N$ (which need not be positive) such that for every composant $C$ of $\widehat{X}, h(C)=\sigma^{N}(C)$.

In [BJK], Block, Jakimovik, and Keesling proved that the Pseudo-isotopy Conjecture implies Ingram's Conjecture. In [BKRS], Block, Keesling, Raines, and Stimac prove that if the turning point of the tent map $f$ is not recurrent, then every homeomorphism of the inverse limit is isotopic to $\sigma^{N}$ for some $N$.

The proofs given here will follow a similar strategy to [BJKK], by first proving that the maps in question satisfy the corresponding version of 
the Pseudo-isotopy Conjecture, and then showing that distinct maps have enough differences in their periodic points in order to complete the proof.

In the remainder of this section, we give the basic definitions and terminology. In Section 2, we cover some basics on itineraries and inverse limits, including the representation of our inverse limit spaces as spaces of biinfinite (two-sided) itineraries. In Section 3, we include a number of miscellaneous results which will be needed for Section 4. In Section 4, we have the main argument, showing that in the inverse limit spaces of interest to us, every homeomorphism switches composants in the same way as some $\widehat{\sigma}^{n}$, where $\widehat{\sigma}$ is the inverse limit shift map. In Section 5 we investigate the periodic points of the maps $\phi_{k, j, \lambda}$ in more detail, showing that if $\lambda_{1}<\lambda_{2}$, then $\phi_{k, j, \lambda_{2}}$ has "more" periodic points than $\phi_{k, j, \lambda_{1}}$, in a way which can be made precise. Section 6 discusses some conjectures and other loose ends.

Definition 1.2. We let $\omega$ denote the set of nonnegative integers, let $\mathbb{Z}$ denote the integers, and let $\mathbb{Z}_{-}$denote the set of negative integers. A sequence will be called respectively infinite, biinfinite, reverse or finite if its domain is respectively $\omega, \mathbb{Z}, \mathbb{Z}_{-}$, or $\{0,1, \ldots, n-1\}$ for some $n \in \omega$. Concatenation of sequences is indicated by juxtaposition in the obvious way (so if $\alpha$ is a finite sequence, then $\alpha \beta$ indicates the sequence $\alpha$ followed by the sequence $\beta$ ). Finite repetitions of the same sequence are indicated by exponents, and infinite repetitions are indicated by a bar, so for example, $10 \overline{0^{3} 1}=100001000100010001 \ldots$ If $\alpha$ is a biinfinite sequence, a "decimal point" is used to mark the position between $\alpha_{-1}$ and $\alpha_{0}$, so for example $\alpha=\overline{0} . \overline{1}$ has $\alpha_{n}=0$ for $n<0$ and $\alpha_{n}=1$ for $n \geq 0$. If $\alpha$ is a finite sequence, $\widehat{\alpha}$ represents the biinfinite sequence $\bar{\alpha} \cdot \bar{\alpha}$.

Definition 1.3. An arc is a space homeomorphic to the closed unit interval $[0,1]$. When we refer to the "interior" of an arc we mean the arc minus its endpoints, without necessarily implying that the "interior" is open in the space containing the arc. A ray is any one-to-one continuous image of the half-open interval $[0,1)$. A tree is a finite union of arcs which contains no simple closed curve. A continuum is a compact connected metric space. A dendrite is a locally-connected, uniquely arcwise-connected continuum. The valence of a point $x$ in a tree $T$ is the number of components of $T \backslash\{x\}$. An endpoint of $T$ is a point of valence 1, and a branching point is a point of valence 3 or more. A star is a tree with no more than one branching point. An $n$-star consists of a center point with $n$ arcs attached at their endpoints. An arc is considered a degenerate 1-star and 2-star. A continuum is decomposable if it can be written as the union of two proper subcontinua, and indecomposable otherwise. If $C$ is a continuum and $c \in C$, then the composant of $c$ is the union of all proper subcontinua containing $c$. 
Definition 1.4. If $X$ is a topological space and $f: X \rightarrow X$ is continuous, then the inverse limit $\widehat{X}$ of $X$ with respect to $f$ is the set of all reverse sequences $\widehat{x}=\left\langle\ldots, x_{-3}, x_{-2}, x_{-1}\right\rangle$ from $X$ such that $f\left(x_{n-1}\right)=$ $x_{n}$ for all $n \in \mathbb{Z}_{-}$, with the topology inherited from the product topology. The shift map $\widehat{f}: \widehat{X} \rightarrow \widehat{X}$ is defined by $\widehat{f}\left(\left\langle\ldots, x_{-3}, x_{-2}, x_{-1}\right\rangle\right)=$ $\left\langle\ldots, f\left(x_{-3}\right), f\left(x_{-2}\right), f\left(x_{-1}\right)\right\rangle=\left\langle\ldots, x_{-2}, x_{-1}, f\left(x_{-1}\right)\right\rangle$.

Definition 1.5. A continuous tree map $f: T \rightarrow T$ is said to be Markov if there is a finite set $P \subseteq T$ containing all branching points and endpoints of $T$ such that $f(P) \subseteq P$ and $f$ is one-to-one on all components of $T \backslash P$. Such a $P$ is called a Markov set for $f$. If such a $P$ exists, there is always a unique minimal such $P$, called the minimal Markov set for $f$. A Markov map $f: T \rightarrow T$ with minimal Markov set $P$ is said to be expansive if for every nonempty open set $U$ there is a positive integer $n$ such that $f^{n}(U) \cap P \neq \emptyset$. A continuous function $f$ on a topological space $X$ is called locally eventually onto if for every nonempty open subset $U$ of $X$ there is a positive integer $n$ such that $X \subseteq f^{n}(U)$. A locally eventually onto Markov map is easily seen to be expansive. If $P$ is a Markov set for $f$, the closure of a component of $T \backslash P$ is called a $P$-basic interval. If $I$ and $J$ are $P$-basic intervals, then we say that $I f$-covers $J$ if $J \subseteq f(I)$. The Markov graph of $f$ with respect to $P$ is the directed graph having $P$-basic intervals as its vertices, with an "arrow" from $I$ to $J$ if and only if $I f$-covers $J$ (abbreviated $I \rightarrow J$ for convenience). A loop of length $n$ in the Markov graph is a sequence $I_{0} \rightarrow I_{1} \rightarrow \cdots \rightarrow I_{n}=I_{0}$ in the Markov graph. A loop is repetitive if it consists of a smaller loop repeated an integer number of times. The incidence matrix of a Markov graph with respect to an enumeration $\left\{I_{1}, \ldots, I_{n}\right\}$ of the $P$-basic intervals is the $0-1$ matrix $B=\left(b_{i j}\right)$ where $b_{i j}=1$ if and only if $I_{i} \rightarrow I_{j}$.

Definition 1.6. Another directed graph related to the Markov graph is also useful. If $[a, b]$ is a $P$-basic interval, then we call $[a, b)$ a $P$-basic ray (with $[a, b)$ and $[b, a)$ considered as distinct $P$-basic rays). The ray graph of $f$ with respect to $P$ will be the set of all $P$-basic rays, with an "arrow" from $[a, b)$ to $[c, d)$ if and only if $[a, b] \rightarrow[c, d]$ in the Markov graph and $f(a)=c$. We note that for every $P$-basic ray $[a, b)$ there is exactly one $P$-basic ray $[c, d)$ such that $[a, b) \rightarrow[c, d)$. Loops are defined the same way as for the Markov graph. A $P$-basic ray is said to be essential if it is part of a loop in the ray graph. The point of $P$ to which an essential ray is attached must be periodic, and for each periodic point $x$ of $P$, the type of $x$ is defined to be the number of essential $P$-basic rays emanating from $x$. Note that points in the same periodic orbit have the same type.

The following result is a minor variation of a well known result. (We adopt the convention that the loop $I_{0} \rightarrow I_{1} \rightarrow I_{2} \rightarrow \cdots \rightarrow I_{n}=I_{0}$ and $I_{1} \rightarrow I_{2} \rightarrow \cdots \rightarrow I_{n}=I_{0} \rightarrow I_{1}$ are considered the same loop.) 
Theorem 1.7. Let $f: T \rightarrow T$ be an expansive Markov tree map. Then there is a natural one-to-one correspondence between nonrepetitive loops in the Markov graph and periodic orbits. If the length of a nonrepetitive loop is not the same as the period of its corresponding periodic orbit, then the loop does not repeat any basic intervals (so there can be only finitely many such loops).

2. Itineraries and inverse limits. In the main examples $f: T \rightarrow T$ of interest to us in this paper, the unique turning point $t$ of $f$ is of valence two. Thus, we can form a partition of $T$ consisting of three pieces, the singleton $\{t\}$ and the two components of $T \backslash\{t\}$. We label $\{t\}$ as $S_{*}$, the component of $T \backslash\{t\}$ containing $f(t)$ is labelled $S_{0}$, and the other component is labelled $S_{1}$. (The turning point $t$ will not be a fixed point of $f$ in cases of interest to us.) The symbols $0,1, *$ correspond to the $R, L, C$ which are often used for maps of the interval. Using this partition, we can define "itineraries" of points of $T$ in the usual way:

Definition 2.1. Let $\mathcal{S}=\left\{S_{0}, S_{1}, S_{*}\right\}$ be the partition of $T$ described above. If $x \in T$, we say that $\iota_{f}(x)$ is the itinerary of $x$ with respect to $f$ if $\iota_{f}(x)$ is a sequence $\alpha$ with domain $\omega$ such that $f^{n}(x) \in S_{\alpha_{n}}$ for all $n \in \omega$. The function $f$ is said to have the unique itinerary property if $x \neq y$ implies $\iota_{f}(x) \neq \iota_{f}(y)$. The kneading sequence of $f$ is the sequence $\iota_{f}(f(t))$.

While the kneading sequence obviously depends on the function $f$, what is not so clear is that the kneading sequence determines the conjugacy class of $f$, if we add the reasonable hypotheses that $f$ satisfies the unique itinerary property, and that no subtree of $T$ contains the orbit of $f$. This follows from the theory of Hubbard Trees (see, e.g., [BS]), or from the classification of dendrite maps with the unique itinerary property (see [Bal]). A brief description of the latter follows. (We restrict the discussion to periodic $\tau$.)

Definition 2.2. We topologize $\{0,1, *\}$ by the basis $\{\{0\},\{1\},\{0,1, *\}\}$, and the itinerary topologies on $\{0,1, *\}^{\omega}$ and $\{0,1, *\}^{\mathbb{Z}}$ are the product topologies thus generated. If $a, b \in\{0,1, *\}$, then we say that $a \approx b$ if either $a=b$ or at least one of $a$ and $b$ is $*$. (That is, the symbol $*$ is thought of as a "wild card" which can stand for either 0 or 1.) If $\alpha$ and $\beta$ are functions with the same domain $D$ and values in $\{0,1, *\}$, we say that $\alpha \approx \beta$ if $\alpha_{n} \approx \beta_{n}$ for all $n \in D$. A periodic sequence $\tau=\overline{\theta *}(\theta$ a finite sequence of 0 's and 1 's) is called acceptable if for all $n \in \omega, \sigma^{n}(\tau) \approx \tau$ implies $\sigma^{n}(\tau)=\tau$. If $\tau$ is acceptable, then a sequence $\alpha$ is called $\tau$-consistent if for all $n \in \omega, \alpha_{n}=*$ if and only if $\sigma^{n+1}(\alpha)=\tau$. A $\tau$-consistent sequence $\alpha$ is called $\tau$-admissible if for all $n \in \omega, \sigma^{n}(\alpha) \approx \tau$ implies $\sigma^{n}(\alpha)=\tau$. The set of all $\tau$-admissible sequences from $\{0,1, *\}^{\omega}$ is called $D_{\tau}$, given the topology inherited from the itinerary topology. $D_{\tau}$ is known to be a dendrite ([Bal, Theorem 2.27]). We 
define $T_{\tau}$ to be the smallest subtree of $D_{\tau}$ containing the orbit of $\tau$ (which is finite in the case under consideration).

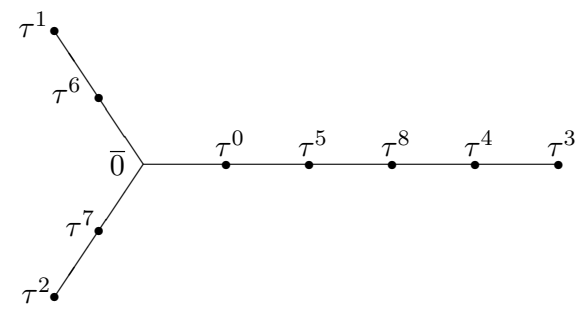

Fig. 2. Diagram of $T_{\tau}, \tau=\overline{00111001 *}$

There are a number of advantages to regarding our tree to be a subset of $D_{\tau}$. One is that the kneading sequence gives us a more convenient way of identifying examples than the parameters $n, k, \lambda$ of $\phi_{n, k, \lambda}$. Also, we will want to also consider examples of tree maps where the tree is not a star, and the kneading sequence $\tau$ gives us a way to describe such maps. Another advantage is that in $D_{\tau}$, points are identified by their itineraries, and thus the points themselves tell us something of their dynamics. This leads to another advantage: points in the inverse limit space can then be identified as biinfinite sequences. This will be useful because points having a $*$ on some coordinate will be points that we are interested in keeping track of.

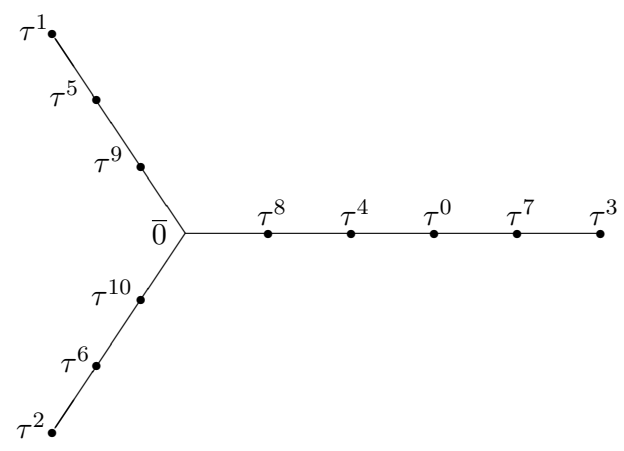

Fig. 3. Diagram of $T_{\tau}, \tau=\overline{0010001000 *}$

Figures 2, 3, and 4 show three examples of shift functions $\sigma: T_{\tau} \rightarrow T_{\tau}$, showing their kneading sequences, the structure of the tree, and the way the main periodic orbits and the branching points map to each other. (For some examples which are not stars, see also Section 6.) Here, the turning point is labelled $\tau^{0}$, its image (the point having itinerary $\tau$ ) is labelled $\tau^{1}$, and so forth up to $\tau^{n-1}$, which maps back to $\tau^{0}$. The branching points are identified by their itineraries. 


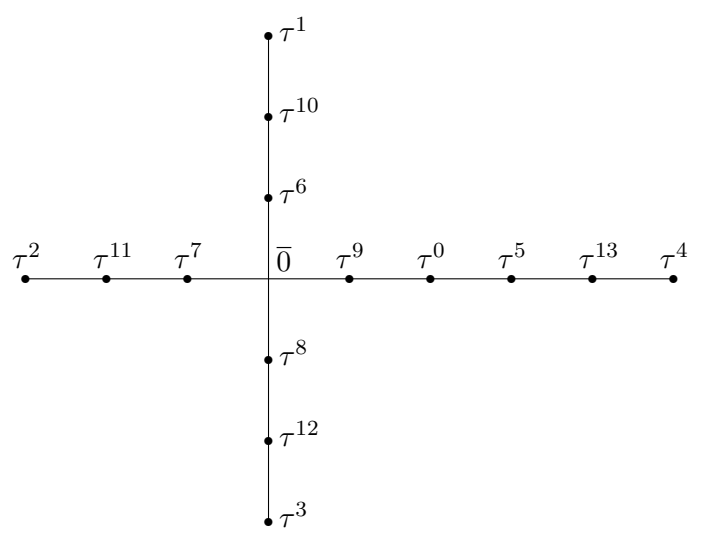

Fig. 4. Diagram of $T_{\tau}, \tau=\overline{0001100000001 *}$

Definition 2.3. If $\alpha$ is a biinfinite sequence, and $n$ is an integer, then $\pi_{n}(\alpha)$ is defined to be the infinite sequence $\beta$ with domain $\omega$ such that $\beta_{i}=\alpha_{n+i}$ for all $i \in \omega$. If $\tau$ is acceptable, $\widehat{D}_{\tau}$ is defined to be the set of all biinfinite sequences $\alpha$ such that $\pi_{n}(\alpha) \in D_{\tau}$ for all $n \in \mathbb{Z}$, with the topology inherited from the itinerary topology on $\{0,1, *\}^{\mathbb{Z}}$. Let $\widehat{\sigma}$ be the shift map on $\widehat{D}_{\tau}$. Similarly $\widehat{T}_{\tau}$ is defined to be the subset of $\widehat{D}_{\tau}$ consisting of all $\alpha$ such that $\pi_{n}(\alpha) \in T_{\tau}$ for all $n \in \mathbb{Z}$. If $\alpha$ is a biinfinite sequence, and $n$ is an integer, then $\varpi_{n}(\alpha)$ is defined to be the reverse sequence $\beta$ with domain $\mathbb{Z}_{-}$ such that $\beta_{i}=\alpha_{n+i}$ for all $i \in \mathbb{Z}_{-}$.

Proposition 2.4 (cf. [BrD, Theorem 2.5]). $\widehat{D}_{\tau}$ is homeomorphic to the inverse limit of $D_{\tau}$ with bonding map $\sigma$, and $\widehat{\sigma}$ is conjugate to the corresponding inverse limit shift map.

Proof. Let $\widehat{X}$ be the inverse limit of $D_{\tau}$ with bonding map $\sigma$, and let $\widehat{f}$ be the corresponding shift map. Define $h: \widehat{D}_{\tau} \rightarrow \widehat{X}$ by $h(\alpha)=$ $\left\langle\ldots, \pi_{-3}(\alpha), \pi_{-2}(\alpha), \pi_{-1}(\alpha)\right\rangle$. Then, since the $\pi_{n}$ 's are clearly continuous, $h$ is easily seen to be a continuous bijection from $\widehat{D}_{\tau}$ to $\widehat{X}$ such that $h \circ \widehat{\sigma}$ $=\widehat{f} \circ h$. Thus, since $\widehat{D}_{\tau}$ is a compact Hausdorff space, $h$ is the desired homeomorphism giving a conjugacy between $\widehat{\sigma}$ and $\widehat{f}$.

Note that the maps $\pi_{n}: \widehat{D}_{\tau} \rightarrow D_{\tau}$ correspond to the natural projections from $\widehat{X}$ to $D_{\tau}$. This leads to the following easy proposition, a minor variation of a well known result.

Proposition 2.5. $\pi_{n} \circ \widehat{\sigma}=\sigma \circ \pi_{n}=\pi_{n+1}$.

Proof. If $\alpha \in \widehat{D}_{\tau}$, then $\pi_{n}(\widehat{\sigma}(\alpha))=\sigma\left(\pi_{n}(\alpha)\right)=\pi_{n+1}(\alpha)=\left\langle\alpha_{n+1}, \alpha_{n+2}\right.$, $\left.\alpha_{n+3}, \ldots\right\rangle$.

In addition to the functions $\pi_{n}$ defined above, there are the projection maps which take $\alpha$ to $\alpha_{n}$, thus mapping $D_{\tau}$ continuously onto the three- 
point connected space $\{0,1, *\}$. Thus, if $\alpha_{n}=0$ and $\beta_{n}=1$, then any connected subset of $D_{\tau}$ containing both $\alpha$ and $\beta$ must also contain a $\gamma$ such that $\gamma_{n}=*$ (since $\{0,1\}$ is not connected). This observation is the motivation behind the following process.

Definition 2.6. Let $\tau \in \mathcal{K}$, and let $\alpha, \beta \in D_{\tau}$. We define $\mu=\mu_{\tau}(\alpha, \beta) \in$ $D_{\tau}$ as follows. We let $n$ be least such that $\alpha_{n} \not \approx \beta_{n}$. (If no such $n$ exists, then $\alpha=\beta$, and we let $\mu=\alpha=\beta$.) For $m<n$ we let $\mu_{m}^{\prime}$ be whichever (or both) of $\left\{\alpha_{m}, \beta_{m}\right\}$ is not $*$. We let $\mu_{n}^{\prime}=*$, and $\mu_{m}^{\prime}=\tau_{m-1-n}$ for $m>n$. Then there is a unique $\mu \in D_{\tau}$ with $\mu \approx \mu^{\prime}$. Then $\mu_{\tau}(\alpha, \beta)$ is in any connected subset of $D_{\tau}$ containing $\alpha$ and $\beta$ (see the proof of [Bal, Theorem 2.25]). If $\alpha$ and $\beta$ are biinfinite sequences such that there is an $N$ such that $\alpha_{n} \approx \beta_{n}$ for all $n<N$, then $\mu_{\tau}(\alpha, \beta)$ can be defined similarly as a biinfinite sequence.

For example, if $\tau=\overline{001 *}, \alpha=1 \overline{0}$, and $\beta=* \tau=* \overline{001 *}$, then $\mu=$ $\mu^{\prime}=100 * \tau$. An example where $\mu \neq \mu^{\prime}$ would be $\tau=\overline{001 *}, \alpha=1001 \overline{0}$, $\beta=10011 \overline{0}$. This would give $\mu^{\prime}=1001 * \tau=1 \tau$, and the initial 1 would then have to be changed to a $*$ to get $\mu=* \tau$ in order to get a $\tau$-admissible element.

The $\mu$ function can be repeatedly applied to locate all points on the arc from $\alpha$ to $\beta$ which contain a $*$. Such iterations will be called the " $\mu$-process" below. Applications of this process to numerous examples provided useful intuition in the initial stages of this research. This process was used in [Bal] to prove that $D_{\tau}$ is arcwise-connected ([Bal, Theorem 2.25]).

Proposition 2.7. Let $\alpha, \beta \in D_{\tau}$ be such that $\alpha_{n} \approx \beta_{n}$ for all $n \leq N$. Let $A$ be the arc in $D_{\tau}$ having $\alpha$ and $\beta$ as endpoints. Then for every $\gamma \in A$ and every $n \leq N, \alpha_{n} \approx \gamma_{n} \approx \beta_{n}$. The same result is true if $\alpha, \beta$ in $\widehat{D}_{\tau}$ are such that $\alpha_{n} \approx \beta_{n}$ for all $n \leq N$.

Proof. This follows from the proof of [Bal, Theorem 2.25].

We end this section with a generalization of a theorem of Brucks and Diamond characterizing the composants of the inverse limit space $([\mathrm{BrD}$, Lemmas $2.8 \& 2.9$, Corollary 2.10]). If $\alpha$ is a function with range $\{0,1, *\}$, then let us call any $\beta$ with range $\{0,1\}$ such that $\alpha \approx \beta$ a substitution of $\alpha$.

Theorem 2.8. Let $\tau=\overline{\theta *}$ be acceptable, let $\widehat{\tau}=\widehat{\theta *}$, and let $\tau^{\prime}$ be the reverse sequence $\varpi_{0}(\widehat{\tau})$. Then $\alpha$ and $\beta$ are in the same composant of $\widehat{D}_{\tau}$ if and only if there is an $n \in \mathbb{Z}$ such that either $\varpi_{n}(\alpha)=\varpi_{n}(\beta)$ or $\varpi_{n}(\alpha)$ and $\varpi_{n}(\beta)$ are both either $\tau^{\prime}$ or a substitution of $\tau^{\prime}$.

Proof. If $n$ is such that $\varpi_{n}(\alpha)=\varpi_{n}(\beta)$, then application of the $\mu$-process gives an arc between $\alpha$ and $\beta$. The same is true if one of $\varpi_{n}(\alpha)$ and $\varpi_{n}(\beta)$ is $\tau^{\prime}$ and the other is a substitution of $\tau^{\prime}$. This gives one direction of the theorem. 
For the other direction, we first prove the following claim.

Claim. No arc of $D_{\tau}$ contains more than one member of the orbit of $\widehat{\tau}$.

Proof of Claim. By contradiction. If there is such an arc, then there is an $\operatorname{arc} A$ in $D_{\tau}$ having endpoints $\gamma$ and $\delta$, both members of the orbit of $\widehat{\tau}$, but no other member of $A$ is in the orbit of $\widehat{\tau}$. Let $p$ be the period of $\tau$. By acceptability of $\tau$, there is an $n$ such that $\gamma_{n} \not \approx \delta_{n}$, and thus $\gamma_{n-i p} \not \approx \delta_{n-i p}$ for all $i \in \omega$. Thus, there are $\eta^{i} \in A$ such that $\eta_{n-i p}^{i}=*$. Then the $\eta^{i}$ 's converge to a member of the orbit of $\widehat{\tau}$ distinct from $\gamma$ and $\delta$, a contradiction.

Now, suppose that $\alpha$ and $\beta$ are members of $D_{\tau}$ such that for every $n \in \mathbb{Z}$, $\varpi_{n}(\alpha) \neq \varpi_{n}(\beta)$ and $\varpi_{n}(\alpha)$ and $\varpi_{n}(\beta)$ are not both $\tau^{\prime}$ or a substitution of $\tau^{\prime}$. Aiming for a contradiction, suppose that $A$ is an arc containing both $\alpha$ and $\beta$. Then there is a sequence $\left\langle n_{i}: i \in \omega\right\rangle$ of integers approaching $-\infty$ such that $\alpha_{n_{i}} \not \approx \beta_{n_{i}}$. By thinning to a subsequence if necessary, we may assume that the $n_{i}$ 's are all in the same congruence class modulo $p$. Then there are $\gamma^{i} \in A$ such that $\gamma_{n_{i}}^{i}=*$, and $\gamma^{i}$ converges to some $\gamma$ in the orbit of $\widehat{\tau}$. Repeating the argument with $\gamma$ and either $\alpha$ or $\beta$ gives the same contradiction as in the claim.

If one compares the statement of this theorem with the similar theorem in $[\mathrm{BrD}]$, one will notice that the present theorem has a phrase ( “ $\ldots$ or $\varpi_{n}(\alpha)$ and $\varpi_{n}(\beta)$ are both either $\tau^{\prime}$ or a substitution of $\left.\tau^{\prime \prime \prime}\right)$ which seems to have no analogue in the Brucks-Diamond paper. This is partly due to the more general situation with the dendrite $D_{\tau}$, and partly due to notation adopted in $[\mathrm{BrD}]$ regarding the kneading sequence.

3. Miscellaneous results. In the families $\phi_{k, j, \lambda}$ defined above, we will be primarily interested in values $k$ and $j<k$ such that $j$ and $k$ are relatively prime. For, suppose that $j$ and $k$ are not relatively prime. Then let $d$ be the greatest common divisor of $j$ and $k$, and it is not difficult to see that for each $m, 0 \leq m \leq d-1$, the set $S_{m}^{\prime}=\bigcup_{i=0}^{k / d} S_{i d+m}$ is a proper subset of the $k$-star $S_{k}$ which is invariant under $\phi_{k, j, \lambda}$, and that the inverse limit of $S_{k}$ with respect to $\phi_{k, j, \lambda}$ is thus decomposable. (In fact, in this case $\phi_{k, j, \lambda}^{d} \mid S_{m}^{\prime}$ is in fact conjugate to $\phi_{k / d, j / d, \lambda^{d}}$.) The other reason we are interested in relatively prime $j$ and $k$ is the following result.

Proposition 3.1. If $k$ and $j$ are relatively prime, then $\phi=\phi_{k, j, \lambda}$ is locally eventually onto.

Proof. Let $U \subseteq S_{k}$ be a nonempty open set. We prove by several stages that there is an $n$ such that $\phi^{n}(U)$ is all of $S_{k}$. Let $c$ be the branching point of $S_{k}$, and let $I_{i}, 0 \leq i \leq k-1$, be the closures of the components of $S_{k} \backslash\{c\}$, as in Definition 1.1. For convenience, think of $I_{i}$ as being indexed by $i \in \mathbb{Z}_{k}$. 
Claim 1. There is a positive integer $n$ such that $c \in \phi^{n}(U)$.

Proof of Claim 1. By contradiction. Suppose $c \notin \phi^{n}(U)$ for all $n \in \omega$. By shrinking $U$ if necessary, we may assume that $U$ is an interval of length $a_{0}>0$. Let $a_{n}$ be the length of the interval $\phi^{n}(U)$, and note that the $a_{n}$ 's are bounded. If $t \notin \phi^{n}(U)$, then $a_{n+1}=\lambda a_{n}$, whereas if $t \in \phi^{n}(U)$, we only know that $a_{n+1} \geq \frac{1}{2} \lambda a_{n}$. Note that since $\phi(t) \in I_{0}$ and $\phi\left(I_{i}\right)=I_{i+1}$ for $0 \leq i \leq k-2, t$ can be in at most every $k$ th $\phi^{n}(U)$, i.e., if $t \in \phi^{n}(U)$, then $t \notin \phi^{i}(U)$ for $n+1 \leq i \leq n+k-1$. Thus, $a_{k n} \geq\left(\lambda^{k} / 2\right)^{n} a_{0}$, which gets arbitrarily large, contradicting that the $a_{n}$ 's are bounded. This completes the proof of Claim 1.

Claim 2. There is a positive integer $m$ such that $\phi^{m}(U)$ contains $I_{i}$ for some $i$.

Proof of Claim 2. Starting with an $n$ such that $c \in \phi^{n}(U)$, let $J$ be a nondegenerate subinterval of $\phi^{n}(U)$ with $c$ as one endpoint. Then the length of $\phi^{i}(J)$ increases by a factor of $\lambda$ with each iteration until we get $t \in \phi^{i}(J)$, which gives $I_{0} \subseteq \phi^{i+1}(J) \subseteq \phi^{n+i+1}(U)$. This completes the proof of Claim 2 .

Let $m<k$, and suppose that a positive integer $n_{m}$ has been found such that $\phi^{n_{m}}(U)$ contains $m$ of the intervals $I_{i}$, say $Z$ is a subset of $\mathbb{Z}_{k}$ of cardinality $m$ such that $I_{i} \subseteq \phi^{n_{m}}(U)$ for each $i \in Z$. Then since $j$ and $k$ are relatively prime, there is a $p \in Z$ such that $p+j \notin Z$ (where the addition is in $\mathbb{Z}_{k}$ ). Then $\phi^{n_{m}+k-p}(U)$ contains $I_{i+k-p}$ for all $i \in Z$, plus it contains a portion of $I_{j}$. Repeating the proof of Claim 2 then gives that for some $i>n_{m}, \phi^{i}(U)$ contains $m+1$ of the intervals $I_{i}$. By induction, we are done.

TheOREM 3.2. Let $f: S \rightarrow S$ be a continuous function on a star having exactly one turning point which is not the branch point of the star, and suppose that $f$ is locally eventually onto. Then there exist $k, j$, and $\lambda$ with $j$ and $k$ relatively prime such that $f$ is conjugate to $\phi_{k, j, \lambda}$.

Proof. Since $f$ being eventually locally onto implies that $f$ is transitive, a theorem of Parry implies that $f$ is conjugate to a function $g: S \rightarrow S$ which is piecewise linear with constant expansion factor equal to some $\lambda>1$ (see $[\mathrm{P}])$. Let $t \in S$ be the turning point of $g$.

CASE 1: $S$ has a branching point $c$ (i.e., $S$ is not an arc). Let $k$ be the valence of $c$. Then $g(c)=c$, since $t$ is the only turning point of $g$. Since $g$ is onto, each endpoint has a $g$-preimage which can only be another endpoint or the turning point. Thus, each endpoint must be in the orbit of the turning point, or the locally eventually onto property would be violated. Therefore, the endpoints can be enumerated as $e_{0}, e_{1}, \ldots, e_{k-1}$ such that $g^{i}(t)=e_{i-1}$, $1 \leq i \leq k$. Let $I_{i}=\left[c, e_{i}\right], 1 \leq i \leq k$. Then, since there are no turning points 
other than $t,[c, t]$ is mapped one-to-one onto $I_{0}$ with expansion factor $\lambda$. Similarly, $I_{i-1}$ is mapped one-to-one onto $I_{i}$ with expansion factor $\lambda, 1 \leq i \leq$ $k-1$. This leaves $\left[t, e_{k-1}\right]$ to be mapped one-to-one with expansion factor $\lambda$ onto some interval having $e_{0}$ as an endpoint. Let $a=g\left(e_{k-1}\right)$. Then $a$ cannot be in $I_{0}$, since then $I_{0}$ would be invariant with respect to $g^{k}$, contradicting that $g$ is locally eventually onto. Similarly, if $a$ is in $I_{j}$ where $j$ and $k$ are not relatively prime, then let $d$ be the greatest common divisor of $j$ and $k$, and we have $\bigcup_{i=0}^{k / d-1} I_{i}$ invariant with respect to $g^{d}$, a contradiction. Thus, we must have $a \in I_{j}$ for some $j$ which is relatively prime to $k$. From this it follows that $g$ is just a rescaled version of $\phi_{k, j, \lambda}$.

CASE 2: $S$ is an arc. Pick $x \in S$ which is not a endpoint, and use the "dog chases rabbit" trick to find a fixed point of $g$, starting at $x$ (vary $x$ [the "dog"] continuously in the direction of $f(x)$ [the "rabbit"], a process that ends when the "dog" catches the "rabbit" at a fixed point). Since any fixed endpoint would be repelling (because $\lambda>1$ ), this process gives us a fixed point $c$ which is not an endpoint of $S$. Now, viewing $c$ as the center point of a star with two endpoints, repeat the argument of Case 1 , with $k=2$ and $j=1$.

Definition 3.3. Extend the definition of valence in the following way. Let $X$ be the inverse limit space of a Markov tree map. A point $x$ of $X$ is said to have valence $n$ if it is the center of some $n$-star but is not the center of any $n+1$-star. Here, the arc is regarded as both a degenerate 1-star (with an endpoint as the center) and as a degenerate 2-star (with a non-endpoint as the center). A point of valence 1 will be called an endpoint, and a point of valence 3 or more will be called a branching point.

Definition 3.4. Let $f: X \rightarrow X$ and let $\widehat{f}: \widehat{X} \rightarrow \widehat{X}$ be the corresponding inverse limit shift map. We say that a periodic point $\widehat{x}$ of $\widehat{f}$ with orbit $\widehat{A}$ corresponds to a periodic point $x$ of $f$ with orbit $A$ if $\pi_{n}(\widehat{A})=A$ for each of the natural projections $\pi_{n}$.

Lemma 3.5. Let $f: T \rightarrow T$ be a locally eventually onto Markov tree map with minimal Markov set $P$, and suppose that $\left\langle C_{n}: n \in \mathbb{Z}_{-}\right\rangle$is a reverse sequence of subtrees of $T$, not all equal to $T$, such that for all $n \in \mathbb{Z}_{-}$, $C_{n} \cap P \neq \emptyset$ and $f\left(C_{n-1}\right)=C_{n}$. Then there is an integer $N$, and there is a single periodic orbit from $P$, enumerated cyclically as $\left\langle x_{n}: n \in \mathbb{Z}_{-}\right\rangle$, such that $x_{n} \in C_{n}$ and $f\left(x_{n-1}\right)=x_{n}$ for all $n$, and such that for all $n \leq N, C_{n}$ is a subset of the union of the essential P-basic rays emanating from $x_{n}$.

Proof. Since $f$ is eventually locally onto, there is a positive integer $m$ such that $f^{m}(I)=T$ for all $P$-basic intervals $I$. Thus, there must be an integer $N$ such that $n \leq N$ implies that $C_{n}$ does not contain a $P$-basic interval, for otherwise all $C_{n}$ 's would be equal to $T$, contradicting the hypothesis. Thus, for $n \leq N, C_{n} \cap P$ is a singleton $\left\{x_{n}\right\}$, and it is easy to see that if 
we define $x_{n}=f^{n-N}\left(x_{N}\right)$ for all $n>N$, then we have $f\left(x_{n-1}\right)=x_{n}$ for all $n$. Fix $n \leq N$, and let $a_{n} \in C_{n}$. Then for $m<n$ there are $a_{m} \in C_{m}$ such that $f\left(a_{m-1}\right)=a_{m}$. Each $a_{m}$ is clearly in a $P$-basic ray emanating from $x_{m}$, from which it is clear that $a_{n}$ must be in an essential $P$-basic ray emanating from $x_{n}$.

TheOREM 3.6. Let $f: T \rightarrow T$ be a locally eventually onto Markov tree map with minimal Markov set $P$, and let $\widehat{f}: \widehat{T} \rightarrow \widehat{T}$ be the corresponding inverse limit shift map. Then every proper subcontinuum of $\widehat{T}$ is either a star, an arc, or a point. No composant of $\widehat{T}$ contains more than one periodic point of $\widehat{T}$. All but finitely many points of $\widehat{T}$ have open neighborhoods which are homeomorphic to a Cantor set cross $\mathbb{R}$. Those points of $\widehat{T}$ which have no such neighborhoods are periodic points of $\widehat{f}$ corresponding to periodic points of $f$ in the set $P$. If $\widehat{x} \in \widehat{T}$ is such a point, corresponding to a point $x \in P$, then $\widehat{x}$ has valence $n$, where $n$ is the type of $x$ in the ray graph of $f$.

Proof. Several variations of this theorem are well known. We give the main outline of the proof. Let $C$ be a proper subcontinuum of $\widehat{T}$. Let $C_{n}=$ $\pi_{n}(C)$. Then $C_{n}$ is a subtree of $T$, and not all $C_{n}$ 's are equal to $T$. Thus, since $f$ is locally eventually onto, the diameter of the $C_{n}$ 's goes to zero as $n$ approaches $-\infty$. Thus, there can be only one sequence $\left\langle x_{n}\right\rangle$ consisting of a single periodic orbit with $f\left(x_{n-1}\right)=x_{n}$ for all $n$ such that $x_{n} \in C_{n}$ for all $n$, and therefore $C$ has no more than one periodic point.

CASe 1: There is an $N$ such that $C_{n} \cap P=\emptyset$ for $n \leq N$. Let $U_{N}$ be the interior of the $P$-basic interval containing $C_{N}$. Then $C_{N} \subseteq U_{N}$. For each $n \leq N$, let $U_{n}=f^{-(N-n)}\left(U_{N}\right)$. Then for $n \leq N, U_{n}$ is the disjoint union of open intervals, each of which maps homeomorphically onto $U_{N}$ via $f^{N-n}$. Then $\pi_{n}^{-1}\left(U_{N}\right)$ contains $C$ and is homeomorphic to the inverse limit of the $U_{n}$ 's $(n<N)$, which in turn is homeomorphic to a Cantor set cross $\mathbb{R}$.

CASE 2: $C_{n} \cap P \neq \emptyset$ for all $n$. Then Lemma 3.5 applies, and there is an integer $N$ and a single periodic orbit from $P$, enumerated cyclically as $\left\langle x_{n}: n \in \mathbb{Z}\right\rangle$, such that $x_{n} \in C_{n}$ and $f\left(x_{n-1}\right)=x_{n}$ for all $n$, and such that for all $n \leq N, C_{n}$ is a subset of the union of the essential $P$-basic rays emanating from $x_{n}$. Let $k$ be the type of the $x_{n}$ 's (which are all in the same orbit and therefore have the same type). Then for $n \leq N$, each $C_{n}$ is a $j$-star for some $j \leq k$, and $f$ maps $C_{n-1}$ homeomorphically onto $C_{n}$, so $C$ is homeomorphic to $C_{n}$. Thus, $C$ is a $j$-star for some $j$. In the other direction, it is easy to get an example where $C$ is a $k$-star. Just let $C_{N}$ be any $k$-star contained in the union of the essential $P$-basic rays emanating from $x_{N}$. Then define $C_{n}$ for $n \leq N$ by backward induction on $n$ by letting $C_{n-1}$ be the component of $f^{-1}\left(C_{n}\right)$ containing $x_{n}$. Then the $C_{n}$ 's define a $k$-star $C \subseteq \widehat{T}$. 
Lemma 3.7. Let $\tau$ be an acceptable and periodic sequence from $\{0,1, *\}$ such that $T_{\tau}$ is a $k$-star for some $k \geq 2$. Let $I, J \subseteq T_{\tau}$ be arcs with interiors not containing the fixed point $\overline{0}$ and let $n$ be a positive integer such that $J \subseteq \sigma^{n}(I)$. Then there is a subarc $I^{\prime}$ of I such that $\sigma^{n} \mid I^{\prime}$ is a homeomorphism from $I^{\prime}$ to $J$.

Proof. We prove the statement by induction on $n$. Suppose that $n=1$. Let $I=[a, b]$. If $I$ does not contain the turning point in its interior, then $\sigma \mid I$ is one-to-one and the lemma is trivial, so assume that $I$ contains the turning point $t$ in its interior. Then $\sigma(I)$ is equal to one of $\sigma([a, t])$ and $\sigma([t, b])$, say by symmetry that $I=\sigma([a, t])$. Then there is an $I^{\prime} \subseteq[a, t]$ such that $\sigma^{n} \mid I^{\prime}$ is a homeomorphism from $I^{\prime}$ to $J$.

Let $n>1$ and suppose that the theorem is true when $n$ is replaced by some $m<n$. Let $K=\sigma^{n-1}(I)$. If $t \notin K$, then the induction hypothesis applied successively to 1 and $n-1$ gives us the desired interval $I^{\prime}$, so suppose that $t \in K$. Then $K$ can be written as the union of finitely many intervals $K_{1}, \ldots, K_{j}$, each containing $t$ as an endpoint. Since $\sigma\left(K_{i}\right)$ contains the fixed point for each $i$, it is easily seen that $J \subseteq \sigma\left(K_{i}\right)$ for some $i$. Thus, by the induction hypothesis, we are done.

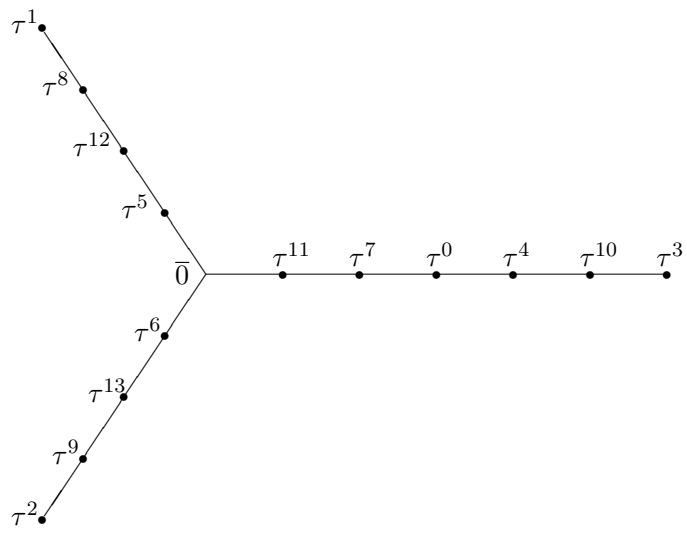

Fig. 5. Diagram of $T_{\tau}, \tau=\overline{0011000001000 *}$

Let $\tau$ be an acceptable and periodic sequence from $\{0,1, *\}$ such that $T_{\tau}$ is a $k$-star for some $k \geq 2$, and let $P$ be the minimal Markov set for this map. Suppose that the branch of the star containing $\tau$ contains at least two elements of the orbit of $\tau$, so that if $E$ is the $P$-basic interval containing $\tau$, then the endpoints of $E$ are $\tau$ and some $\tau^{i}=\sigma^{i-1}(\tau)$ for some $i$. In most cases there will be an integer $m$ such that $\sigma^{m}(\tau)$ and $\sigma^{m}\left(\tau^{i}\right)$ are in different components of $T_{\tau} \backslash\{\overline{0}\}$, but this will not always be true (see Figure 5 for a counterexample). In the general case, we can define an equivalence relation 
$\sim$ on the orbit of $\tau$ by $x \sim y$ if and only if for every $m \in \omega, \sigma^{m}(x)$ and $\sigma^{m}(y)$ are in the same component of $T_{\tau} \backslash\{\overline{0}\}$, and the following proposition is easily seen to hold.

Proposition 3.8. If $\tau$ has period $n$ and $r$ is the number of $\sim$-equivalence classes, then $r>k, n$ is divisible by $r$, and $\sigma^{i}(\tau) \sim \sigma^{j}(\tau)$ if and only if $j-i$ is divisible by $r$.

Proof. $\tau^{0}=* \tau, \tau, \tau^{2}=\sigma(\tau), \ldots, \tau^{k}=\sigma^{k-1}(\tau)$ are easily seen to all be in different equivalence classes, so $r>k$. Let $J_{0}, J_{1}, \ldots, J_{k-1}$ be the components of $T_{\tau} \backslash\{\overline{0}\}$. Define a sequence $\alpha$ by $\alpha_{i}=j$ if and only if $\sigma^{i}(\tau) \in J_{j}$. Then $r$ is the least positive integer such that $\alpha_{i+r}=\alpha_{i}$ for all $i$, from which the rest easily follows.

Theorem 3.9. Let $\tau$ be an acceptable and periodic sequence from $\{0,1, *\}$ with period $n$, such that $\sigma: T_{\tau} \rightarrow T_{\tau}$ is locally eventually onto and $T_{\tau}$ is a $k$-star for some $k \geq 2$. If $P$ is the minimal Markov set of $T_{\tau}$, and $E$ is the $P$-basic interval containing $\tau$, then there are two intervals $I, J \subseteq E$, with $I$ and $J$ intersecting in a single point $c$, and a positive integer $m \leq n-1$ such that $\sigma^{m} \mid I$ (respectively, $\sigma^{m} \mid J$ ) is a homeomorphism from $I$ (respectively, $J$ ) onto $[\overline{0}, \tau]$, with $\sigma^{m}(c)=\tau$.

Proof. Let $E=[\tau, a]$. Let $\tau^{0}=* \tau$ and $\tau^{i}=\sigma^{i-1}(\tau), 1 \leq i \leq n-1$. By Theorem 3.2, $\sigma$ is conjugate to some $\phi_{k, j, \lambda}$ with $k$ and $j$ relatively prime. Let $I_{0}, I_{1}, \ldots, I_{k-1}$ be as in Definition 1.1. Note in particular that $I_{k-1}$ contains the branching point $\overline{0}$, the turning point $\tau^{0}$, the point $1 \overline{0}$ (a preimage of the branching point) and $\tau^{k}$, in that order. Note also that the interval $[\overline{0}, 1 \overline{0}]$ maps via $\sigma$ from $\overline{0}$ out to $\tau$ and then back to $\overline{0}$, so that it is sufficient to find an interval $I \subseteq E$ and an $m \leq n-2$ such that $\sigma^{m} \mid I$ is a homeomorphism onto $[\overline{0}, 1 \overline{0}]$. By Lemma 3.7 , it suffices to find an $m \leq n-2$ such that $\sigma^{m}(E)$ contains both $\overline{0}$ and $1 \overline{0}$.

Claim. If $x, y \in T_{\tau} \backslash\{\overline{0}\}$ are such that $x$ and $y$ are in different $I_{j}$ 's, but $\sigma(x)$ and $\sigma(y)$ are in the same $I_{j}$, then one of $x$ and $y$ is in $\left[1 \overline{0}, \tau^{k}\right]$.

Proof of Claim. Outside of $\left[1 \overline{0}, \tau^{k}\right]$, points of $I_{j}$ map to $I_{j+1}$ (where $j+1$ is modulo $k$ ).

CASE 1: $a$ is the branching point $\overline{0}$. Then $E$ maps via $\sigma^{k-1}$ one-to-one onto $\left[\overline{0}, \tau^{k}\right] \supseteq[\overline{0}, 1 \overline{0}]$, and $k-1 \leq n-2$.

CASE 2: $a=\tau^{i}$ for some $i$, and $a \nsim \tau$. Then there is an $m<n$ such that $\sigma^{m}(\tau)$ and $\sigma^{m}\left(\tau^{i}\right)$ are in different $I_{j}$ 's. Fix the largest such $m$. Since $\sigma^{n}(\tau)=\tau$ and $\sigma^{n}\left(\tau^{i}\right)=\tau^{i}$ are both in $I_{0}, \sigma^{n-1}(\tau)=\tau^{0}$ and $\sigma^{n-1}\left(\tau^{i}\right)=\tau^{i-1}$ must both be in $I_{k-1}$ (the only $I_{j}$ having points which map to $I_{0}$ ), and thus $m \leq n-2$. Since $\sigma^{m}(\tau)$ and $\sigma^{m}\left(\tau^{i}\right)$ are in different $I_{j}$ 's, $\overline{0} \in \sigma^{m}(E)$. By the claim, one of $\sigma^{m}(\tau)$ and $\sigma^{m}\left(\tau^{i}\right)$ is in $\left[1 \overline{0}, \tau^{k}\right]$, so $1 \overline{0} \in \sigma^{m}(E)$. 
CASE 3: $a=\tau^{i}$ for some $i$, and $a \sim \tau$. Let $r$ be the number of $\sim-$ equivalence classes, and let $s=n / r$.

SubcASE 3a: $\overline{0} \in \sigma^{n-r}(E)$. Then since $\overline{0}$ is a fixed point, $\overline{0} \in \sigma^{m}(E)$ for all $m \geq n-r$. Let $m=n-r+k-1$. Then since $r>k, m \leq n-2$. Now, $\tau^{k} \in\left[1 \overline{0}, \tau^{k}\right]$, so since $\tau^{n-r+k} \sim \tau^{k}, \sigma^{m}(\tau)=\tau^{n-r+k} \in\left[1 \overline{0}, \tau^{k}\right]$, and thus $1 \overline{0} \in \sigma^{m}(E)$.

Subcase 3 b: $\overline{0} \notin \sigma^{n-r}(E)$. Then for each $u, 1 \leq u \leq n-r=(s-1) r$, there is a $P$-basic interval contained in $\sigma^{u}(E)$ which is not contained in any $\sigma^{v}(E), 0 \leq v \leq u-1$, for otherwise $\sigma$ would map $\bigcup_{u=0}^{n-r} \sigma^{u}(E)$ into itself, contradicting that $\sigma$ is locally eventually onto. Thus $\bigcup_{u=0}^{s-1} \sigma^{u r}(E)$ must contain at least $s$ distinct $P$-basic intervals (thus containing at least $s+1$ distinct points), and therefore contains a point which is not $\sim$-equivalent to $\tau$. Thus, there is a $u, 1 \leq u \leq s-1$, such that $\sigma^{u r}(E)$ contains a point $\tau^{w}$ not $\sim$-equivalent to $\tau$ (and therefore not $\sim$-equivalent to $\tau^{u r+1}$ ). There must be a $v, 0 \leq v \leq r-1$, such that $\tau^{u r+1+v}$ and $\tau^{w+v}$ are in different $I_{j}$ 's but such that $\tau^{u r+2+v}$ and $\tau^{w+v+1}$ are in the same $I_{j}$. Let $m=u r+v$. Then since $\tau^{w}$ and $\tau^{u r+1}$ are both in $I_{0}, \tau^{w+r}$ and $\tau^{u r+1+r}$ are both in $I_{0}$, and therefore $\tau^{w+r-1}$ and $\tau^{u r+r}$ are both in $I_{k-1}$. Thus $v \leq r-2$ and $m \leq n-2$. The rest of the argument is as in Case 2.

Definition 3.10. Suppose that $\sigma: T \rightarrow T$ is a Markov tree map with Markov set $P$. Let $\mathcal{I}$ be the set of $P$-basic intervals, let $\mathcal{I}^{\prime}$ be a set of symbols, two for each $P$-basic interval $I$, one symbol for $I$ and another for $I^{-1}$ (representing the same interval in the opposite orientation), and let $\mathcal{I}^{*}$ be the set of all words in symbols from the symbols in $\mathcal{I}^{\prime}$. Note that we do not allow "cancellation" between $I$ and $I^{-1}$ here, so $I_{0} I_{1} I_{1}^{-1}$ and $I_{0}$ are distinct words. We define the inverse $w^{-1}$ of a word $w$ by the obvious recursive rules $\left(a^{-1}\right)^{-1}=a$ and $(a b)^{-1}=b^{-1} a^{-1}$. A function $F^{*}$ with domain $\mathcal{I}$ with values in $\mathcal{I}^{*}$ will be called a substitution. A substitution $F^{*}$ is extended to a function (also called $F^{*}$ ) from $\mathcal{I}^{*}$ into itself in the obvious way by the recursive formulas $F^{*}\left(a^{-1}\right)=\left(F^{*}(a)\right)^{-1}$ and $F^{*}(a b)=F^{*}(a) F^{*}(b), a, b$ $\in \mathcal{I}^{*}$. Given a Markov tree map $\sigma$ with Markov set $P$ and $P$-basic intervals $\left\{I_{0}, I_{1}, \ldots, I_{m-1}\right\}$, each of which has been assigned an orientation, define a substitution $B^{*}$, the substitution induced by $\sigma$ (and by the orientation), by letting $B^{*}\left(I_{j}\right)=I_{i_{0}}^{e_{0}} I_{i_{1}}^{e_{1}} \cdots I_{i_{n-1}}^{e_{n-1}}$, where $I_{i_{0}}, I_{i_{1}}, \ldots, I_{i_{n-1}}$ are (in order) the $P$-basic intervals which are $\sigma$-covered by $I_{j}$, and $e_{0}, e_{1}, \ldots, e_{n-1}$ are either 1 or -1 to indicate whether the orientation of each covering is positive or negative.

Repeated substitutions give a simple way of calculating the image under $\sigma^{n}$ of any basic interval. If $I$ is a $P$-basic interval, then $\left(B^{*}\right)^{n}(I)$ is a word giving the image of $I$, showing exactly how the interval is covered, and what 
the repetitions are if $\sigma^{n} \mid I$ is not one-to-one. Thus, substitutions give a useful way of calculating the exact value of $m$ in Theorem 3.9. Starting with the word $w_{0}=E$, where $E$ is a $P$-basic interval containing $\tau$, one forms $w_{m}=$ $\left(B^{*}\right)^{m} E$ for each positive integer $m$ of interest, to see if a subword appears indicating a path from $\overline{0}$ to $\tau$ and back to $\overline{0}$. For example, in the example given in Figure 3, let the $P$-basic intervals be oriented outward from the branching point $\overline{0}$, with $I_{j}$ having "finishing" point $\tau_{j}$. (So, for example, $I_{1}$ goes from $\tau_{5}$ to $\tau_{1}$, and $I_{8}$ goes from $\overline{0}$ to $\tau_{8}$ ). Then, starting with $w_{0}=I_{1}$, we get, in successive substitutions, $w_{1}=I_{2}, w_{2}=I_{3}, w_{3}=I_{4}, w_{4}=I_{5}, w_{5}=I_{6}$, $w_{6}=I_{7}, w_{7}=I_{1}^{-1} I_{5}^{-1} I_{9}^{-1} I_{8}, w_{8}=I_{2}^{-1} I_{6}^{-1} I_{10}^{-1} I_{9}, w_{9}=I_{3}^{-1} I_{7}^{-1} I_{0}^{-1} I_{4}^{-1} I_{8}^{-1} I_{10}$, and $w_{10}=I_{4}^{-1} I_{8}^{-1} I_{9} I_{5} I_{1} I_{1}^{-1} I_{5}^{-1} I_{9}^{-1} I_{8} I_{4} I_{0}$. In the last of these words, we see the subword $I_{9} I_{5} I_{1} I_{1}^{-1} I_{5}^{-1} I_{9}^{-1}$, indicating a path from $\overline{0}$ to $\tau$ and back to $\overline{0}$. Since the period in this case is 11 , this is one of numerous examples in which the number of applications of the substitution $B^{*}$ needed to get the desired subword is one less than the period. Thus, we have shown that the $m \leq n-1$ of the theorem cannot be improved. Using this method, we can also show that the theorem cannot be extended from stars to general trees (for example, the map shown by Figure 9).

We now want to single out the class of functions on which the main argument in Section 4 will be carried out. While our main interest will be the maps $\phi_{k, j, \lambda}$, we wish to also include other tree maps for which the main argument also applies.

Definition 3.11. We say that $\tau$ is in class $\mathcal{K}$ if:

(1) $\tau$ is acceptable, with the range of $\tau$ equal to $\{0,1, *\}$.

(2) $\tau$ is periodic with period $n_{0}$.

(3) $\sigma: T_{\tau} \rightarrow T_{\tau}$ is eventually locally onto.

(4) $\tau$ begins with at least $k-1 \geq 2$ zeros. Equivalently, $\overline{0}$ is a branching point of valence $k \geq 3$.

(5) Branching points of $T_{\tau}$ other than $\overline{0}$, if any, are periodic with valence different from $k$.

(6) $\tau^{k+1}=\sigma^{k}(\tau)$ is in a different component of $T_{\tau} \backslash\{\overline{0}\}$ than $\tau$.

(7) If $P$ is the minimal Markov set of $\sigma: T_{\tau} \rightarrow T_{\tau}$, and $E$ is the $P$-basic interval containing $\tau$, then there are two intervals $I, J \subseteq E$, with $I$ and $J$ intersecting in a point $c$, such that for some $m \leq n_{0}, \sigma^{m} \mid I$ (respectively, $\sigma^{m} \mid J$ ) is a homeomorphism from $I$ (respectively, $J$ ) onto $[\overline{0}, \tau]$, with $\sigma^{m}(c)=\tau$.

The restriction that the range of $\tau$ is equal to $\{0,1, *\}$ guarantees that the turning point of $\sigma \mid T_{\tau}$ is not a branching point. In this paper, we only consider the case where the turning point is periodic, which is equivalent to $\tau$ being periodic. The condition that $\sigma: T_{\tau} \rightarrow T_{\tau}$ is an eventually locally onto tree map guarantees that the inverse limit space is indecomposable. Condition 
(4) guarantees that the point in the inverse limit corresponding to $\overline{0}$ has valence at least 3 . While we are mainly interested in the case when $T_{\tau}$ is a $k$-star with $k \geq 3$, conditions (5) through (7) isolate properties which are true in the star case, but will be enough for the main argument to go through. Condition (5) is a simple way of guaranteeing that any homeomorphism of the inverse limit space will have to fix $\overline{0}$. Condition (6) guarantees that $1 \overline{0}$, the other preimage of the fixed point $\overline{0}$, is between $\tau^{0}$ and $\tau^{k}$. It is used below in Lemma 4.17 and Proposition 4.19. Condition (7), which is slightly weaker than what was proved for stars in Theorem 3.9 (with $n-1$ being replaced by $n$ here), is used below in Lemma 4.35 .

Proposition 3.12. Let $\phi=\phi_{k, j, \lambda}$ for some relatively prime $j$ and $k \geq 3$, and assume that the turning point has a periodic orbit. Then there is a $\tau \in \mathcal{K}$ such that $\phi$ is conjugate to $\sigma: T_{\tau} \rightarrow T_{\tau}$.

Proof. Let $S$ be the domain of $\phi$ and let $t$ be the turning point. Let $S_{*}=\{t\}$ and let $S_{0}$ and $S_{1}$ be the components of $S \backslash\{t\}$, with $S_{0}$ containing the branching point of $S$. Let $\tau$ be the kneading sequence (i.e., the itinerary of $\phi(t))$ with respect to the partition $\left\{S_{*}, S_{0}, S_{1}\right\}$. Then (1), (2), (4), and (5) in the definition of $\mathcal{K}$ are immediate, (3) follows from Theorem 3.1. (6) follows from Theorem 3.2 , and (7) follows from Lemma 3.9.

\section{The main argument}

Definition 4.1. Throughout this section, the following items will be fixed. We let $\tau=\overline{\theta *} \in \mathcal{K}$, with $P$ the minimal Markov set for $\sigma: T_{\tau} \rightarrow T_{\tau}$, and we abbreviate $T=T_{\tau}$. Let $n_{0}$ be the period of $\tau$, let $k \geq 3$ be such that $\tau$ begins with $0^{k-1} 1$, and let $O$ be the orbit of $\tau$ with respect to $\sigma$, with $\tau^{j}$ abbreviating $\sigma^{j-1}(\tau)=\sigma^{j}(* \tau)$ (so that $\tau^{0}$ is the turning point, and $\left.\tau^{1}=\tau\right)$. Let $\mathcal{I}=\left\{I_{0}, I_{1}, \ldots, I_{n_{1}-1}\right\}$ be a fixed enumeration of the $P$-basic intervals (where $n_{1} \geq n_{0}$ is the number of such intervals), and we assume that each member of $\mathcal{I}$ has been assigned an orientation. Let $B$ be the incidence matrix of the Markov graph with respect to this enumeration of $\mathcal{I}$. Let $\mathcal{I}^{\prime}=\mathcal{I} \cup\left\{I^{-1}: I \in \mathcal{I}\right\}$ be a set of symbols for the basic intervals and their "inverses", and let $\mathcal{I}^{*}$ be the set of all words in symbols from $\mathcal{I}^{\prime}$. Let $B^{*}$ be the substitution corresponding to $\sigma$ and the fixed orientation of $P$-basic intervals, where for each $P$-basic interval $I, B^{*}(I)$ is the word (including orientation) describing the image of $I$ under $\sigma$.

We let $\widehat{\sigma}: \widehat{T} \rightarrow \widehat{T}$ be the corresponding inverse limit map, with $\widehat{\tau}=\widehat{\theta *}$ and $\widehat{\tau}^{j}=\widehat{\sigma}^{j-1}(\widehat{\tau})$. Let $\widehat{P}$ consist of all $\widehat{\sigma}$-periodic members of $\widehat{T}$ corresponding to members of $P$. In particular, $\left\{\widehat{\tau}^{j}: 0 \leq j \leq n_{0}-1\right\} \cup\{\widehat{0}\} \subseteq \widehat{P}$ (with equality in the case where $T$ is a star). Let $C(\widehat{x})$ be the composant of $\widehat{T}$ containing $\widehat{x}$ and let $\left\{R_{i}: i \in \mathbb{Z}_{k}\right\}$ be the maximal rays of $C(\widehat{0})$ starting 
at $\widehat{0}$, enumerated in such a way that $\widehat{\sigma}\left(R_{i}\right)=R_{i+1}$ (with addition in $\mathbb{Z}_{k}$ ), and $R_{0}$ contains the point $\overline{0} * . \tau$.

For $p \in \mathbb{Z}$, let $\Phi_{p}=\pi_{p}^{-1}(P)$, let $\Psi_{p}=\pi_{p}^{-1}(O)$, let $\Psi_{p, i}=\pi_{p}^{-1}\left(\tau^{i}\right)$, and let $\Psi^{*}=\bigcup_{p \in \mathbb{Z}} \Psi_{p}$. For $\widehat{x} \in \Psi^{*}$, we let $C(\widehat{x})$ be the least integer $n$ such that $x_{n}=*$, with $C\left(\widehat{\tau}^{j}\right)=-\infty$. For $\widehat{x} \in \Psi_{p}$, the p-level of $\widehat{x}$, denoted $L_{p}(\widehat{x})$, is given by $L_{p}(\widehat{x})=p-C(\widehat{x})$, with $L_{p}\left(\widehat{\tau}^{j}\right)=\infty$.

In our view of the elements of $\widehat{T}$ as biinfinite itineraries, $L_{p}(\widehat{x})$ is the number of coordinates prior to the $p$ th coordinate where the first $*$ appears. A couple of differences between this definition and the treatment of [K1], $[\mathrm{K} 2]$, and [BJKK] should be noted. $\Phi_{p}$ is defined here for all integers, and not just multiples of $n_{0}$. Also, we are allowing the possibility that $L_{p}(\widehat{x})$ is negative. For example, if $\tau=\overline{001000 *}$, then $\overline{0} .0 * \tau \in \Psi_{0}$, and $L_{0}(\overline{0} .0 * \tau)=-1$.

Proposition 4.2. The following simple relationships hold between the $\Phi_{p}$ 's and the $\Psi_{p}$ 's:

(1) $\Psi_{p} \subseteq \Phi_{p}$.

(2) $\Phi_{p} \subseteq \Phi_{p+1}$.

(3) $\widehat{\sigma}\left(\Phi_{p+1}\right)=\Phi_{p}$.

(4) $\Psi_{p} \subseteq \Psi_{p+1}$.

(5) $\widehat{\sigma}\left(\Psi_{p+1}\right)=\Psi_{p}$.

Proof. Since $O \subseteq P,(1)$ is trivial. To see (2), note that if $\widehat{x} \in \Phi_{p}$, then $\pi_{p}(\widehat{x}) \in P$, and therefore $\pi_{p+1}(\widehat{x})=\sigma\left(\pi_{p}(\widehat{x})\right) \in P$, so $\widehat{x} \in \Phi_{p+1}$.

For (3), note that $\widehat{x} \in \widehat{\sigma}\left(\Phi_{p+1}\right)$ iff $\widehat{\sigma}^{-1}(\widehat{x}) \in \Phi_{p+1}$ iff $\pi_{p+1}\left(\widehat{\sigma}^{-1}(\widehat{x})\right) \in P$ iff $\pi_{p}(\widehat{x}) \in P$ iff $\widehat{x} \in \Phi_{p}$.

(4) is similar to (2), and (5) is similar to (3).

Proposition 4.3. Let $A \subseteq \widehat{T}$ be an arc, and let $p \in \mathbb{Z}$. Then $\pi_{p} \mid A$ is one-to-one if and only if for every $\widehat{x} \in A \cap \Psi^{*}$ which is not an endpoint of $A$, $C(\widehat{x}) \geq p$.

Proof. $(\Rightarrow)$ We prove the contrapositive. Suppose that $\widehat{x} \in A \cap \Psi^{*}$ is a non-endpoint of $A$ such that $C(\widehat{x})=q<p$. Then $\pi_{q+1}(\widehat{x})=\tau$, which is an endpoint of $T$. Thus, $\pi_{q+1} \mid A$ (and therefore $\pi_{p} \mid A$ ) is not one-to-one at $\widehat{x}$.

$(\Leftarrow)$ We prove the contrapositive. Suppose that $\pi_{p} \mid A$ is not one-to-one, and let $\widehat{x}, \widehat{y} \in A$ be distinct such that $\pi_{p}(\widehat{x})=\pi_{p}(\widehat{y})$. Then there is a $q<p$ such that $\left\{x_{q}, y_{q}\right\}=\{0,1\}$, and thus there is a $\widehat{z}$ between $\widehat{x}$ and $\widehat{y}$ such that $z_{q}=*$.

Corollary 4.4. Let $A=[\widehat{x}, \widehat{y}] \subseteq \widehat{T}$ be an arc, let $p \in \mathbb{Z}$, and let $L=\max \left\{L_{p}(\widehat{z}): \widehat{z} \in(\widehat{x}, \widehat{y}) \cap \Psi_{p}\right\}$. Then $\pi_{p-L} \mid A$ is one-to-one.

Proposition 4.5. Let $1 \leq j \leq n_{0}-1$ be such that $\pi_{p}(\widehat{x})=\tau^{j}$ is an endpoint of $T$. Then $L_{p}(\widehat{x}) \geq j$. 
Proof. Note that every endpoint of $T$ is of the form $\tau^{i}$ for some $i$ with $1 \leq i \leq n_{0}-1$. Since $\tau^{j}$ is an endpoint of $T$, it has only one preimage with respect to $\sigma$, namely $\tau^{j-1}$, so we must have $\pi_{p-1}(\widehat{x})=\tau^{j-1}$. Similarly, if $j-1>0$, then $\tau^{j-1}$ is also an endpoint of $T$, and thus $\pi_{p-2}(\widehat{x})=\tau^{j-2}$. Proceeding by induction, we get $\pi_{p-j}(\widehat{x})=\tau^{0}=* \tau$, and thus $L_{p}(\widehat{x}) \geq j$.

Definition 4.6. Let $A \subseteq \widehat{T}$ be an arc. We say that $A$ is a $p$-arc if it has endpoints in $\Phi_{p}$ and does not contain any branching point of $\widehat{T}$ in its "interior". Note that if $q \geq p$, then every $p$-arc is also a $q$-arc. A $p$-arc is oriented if it has one of its endpoints distinguished as the "starting" point and the other as the "finishing" point. Unless otherwise stated, we will assume that $p$-arcs in the composant $C(\widehat{0})$ are oriented in the direction of the rays $R_{i}$. An arc in $\widehat{T}$ with endpoints $\widehat{x}$ and $\widehat{y}$ will be denoted $[\widehat{x}, \widehat{y}]$ (or $[\widehat{y}, \widehat{x}]$ ), and an oriented arc with initial point $\widehat{x}$ and final point $\widehat{y}$ will be denoted $\overrightarrow{[}, \widehat{y}]$.

For each oriented $p$-arc $A$ we define the $p$-shape of $A$, denoted $S_{p}(A)$, to be a word from $\mathcal{I}^{*}$, by induction on the number of elements of $A \cap \Phi_{p}$. If $A \cap \Phi_{p}$ has two elements, then $\pi_{p}(A)$ will be a $P$-basic interval $I$, and we define $S_{p}(A)$ to be either $I$ or $I^{-1}$, depending on whether $\pi_{p} \mid A$ is orientation preserving or orientation reversing according to the fixed orientation of $P$-basic intervals mentioned above. If $A \cap \Phi_{p}$ has $j>2$ points, and $S_{p}\left(A^{\prime}\right)$ has already been defined for all oriented $p$-arcs such that $A^{\prime} \cap \Phi_{p}$ has fewer than $j$ points, then write $A=C \cup D$ for oriented $p$-basic arcs $C$ and $D$ intersecting at a point, with $A$ and $C$ having the same initial point, and $A$ and $D$ having the same final point. We then let $S_{p}(A)$ be the concatenation $S_{p}(C) S_{p}(D)$, which is clearly independent of how $A$ was divided. Roughly, as $\widehat{x}$ proceeds across the $\operatorname{arc} A$ (in the direction of orientation), $S_{p}(A)$ describes the path taken by $\pi_{p}(\widehat{x})$, telling which $P$-basic intervals are covered, and in which order and direction.

For each $p$-arc $A$ (oriented or unoriented), we define the $p$-weight of $A$, denoted $W_{p}(A)$, to be a integer-valued vector with $n_{1}$ coordinates, defined by induction on the number of elements of $A \cap \Phi_{p}$. If $A \cap \Phi_{p}$ has two elements, and $\pi_{p}(A)=I_{n}$, then $W_{p}(A)$ has a 1 in the $n$th coordinate and a 0 elsewhere. If $A \cap \Phi_{p}$ has $j>2$ points, and $S_{p}\left(A^{\prime}\right)$ has already been defined for all $p$-basic arcs such that $A^{\prime} \cap \Phi_{p}$ has fewer than $j$ points, then write $A=C \cup D$ for $p$-arcs $C$ and $D$ intersecting at a point in $\Phi_{p}$, and let $W_{p}(A)=W_{p}(C)+W_{p}(D)$. Roughly, $W_{p}(A)$ tells how many times $\pi_{p} \mid A$ covers each $P$-basic interval.

Clearly, $W_{p}(A)$ is easily recoverable from $S_{p}(A)$ : the $n$th coordinate of $W_{p}(A)$ is just the number of times $I_{n}$ appears in $S_{p}(A)$ plus the number of times $I_{n}^{-1}$ appears in $S_{p}(A)$.

A $p$-arc $[\widehat{x}, \widehat{y}]$ is said to be $p$-symmetric if there is a point $\widehat{v} \in[\widehat{x}, \widehat{y}] \cap \Phi_{p}$ such that $\overrightarrow{[\widehat{v}, \widehat{x}]}$ and $\overrightarrow{[\widehat{v}, \widehat{y}]}$ have the same $p$-shape. In that case, $\widehat{v}$ is said to be the center of the arc $[\widehat{x}, \widehat{y}]$. 
We note that our $p$-symmetric arcs are roughly the same as what were called p-pseudosymmetric arcs in [K1], [K2], and [BJKK].

Proposition 4.7. The following are equivalent for two oriented arcs $A$ and $C$.

(1) $A$ and $C$ have the same p-shape.

(2) There exists an orientation preserving homeomorphism $f: A \rightarrow C$ such that for every $\widehat{x} \in A, \pi_{p}(\widehat{x})=\pi_{p}(f(\widehat{x}))$.

(3) There is a bijection $f: A \cap \Phi_{p} \rightarrow C \cap \Phi_{p}$ which is order preserving with respect to the orientations of $A$ and $C$ such that for every $\widehat{x} \in A \cap \Phi_{p}$, $\pi_{p}(\widehat{x})=\pi_{p}(f(\widehat{x}))$.

Proof. Suppose that (3) holds. Let $A \cap \Phi_{p}=\left\{\widehat{a}_{0}, \widehat{a}_{1}, \ldots, \widehat{a}_{n}\right\}$ and let $A_{i}=\left[\widehat{a}_{i-1}, \widehat{a}_{i}\right]$ for $1 \leq i \leq n$. Similarly, let $C \cap \Phi_{p}=\left\{\widehat{c}_{0}, \widehat{c}_{1}, \ldots, \widehat{c}_{n}\right\}$ and let $A_{i}=\left[\widehat{c}_{i-1}, \widehat{c}_{i}\right]$ for $1 \leq i \leq n$. Then (3) clearly implies that $\pi_{p}\left(\widehat{a}_{i}\right)=\pi_{p}\left(\widehat{c}_{i}\right)$ for $0 \leq i \leq n$ and $\pi_{p}\left(A_{i}\right)=\pi_{p}\left(C_{i}\right)$ for $1 \leq i \leq n$. Define $f: A_{i} \rightarrow C_{i}$ by $f(\widehat{x})=\left(\pi_{p} \mid C_{i}\right)^{-1}\left(\pi_{p}(\widehat{x})\right)$ for $\widehat{x} \in A_{i}$. Then $f$ is well defined on all of $A$ (with $\left.f\left(\widehat{a}_{i}\right)=\widehat{c}_{i}\right)$, and satisfies (2). Thus, (3) implies (2). (2) $\Rightarrow(3)$ and $(1) \Leftrightarrow(3)$ are trivial.

Proposition 4.8. Let $A=\overrightarrow{[\widehat{x}, \widehat{y}]}$ be an oriented $p$-arc, and let $\left.A^{\prime}=\overrightarrow{[\widehat{y}, \widehat{x}}\right]$ be the same arc oriented in the opposite orientation. Then the following are equivalent.

(1) A is p-symmetric.

(2) There is a $w \in \mathcal{I}^{*}$ such that $S_{p}(A)=w^{-1} w$.

(3) $S_{p}(A)=S_{p}\left(A^{\prime}\right)$.

(4) If $A \cap \Psi_{p}$ is enumerated as $\left\{\widehat{x}_{0}, \widehat{x}_{1}, \ldots, \widehat{x}_{n}\right\}$ (in order along $A$ ), then $n$ is even, and for all $i, 0 \leq i \leq n$, we have $\pi_{p}\left(\widehat{x}_{i}\right)=\pi_{p}\left(\widehat{x}_{n-i}\right)$.

(5) There is an orientation reversing homeomorphism $h$ of $A$ such that $\pi_{p}(h(\widehat{a}))=\pi_{p}(\widehat{a})$ for all $\widehat{a} \in A$.

(6) There are a point $\widehat{v} \in A$ and homeomorphisms $f:[0,1] \rightarrow[\widehat{v}, \widehat{x}]$ and $g:[0,1] \rightarrow[\widehat{v}, \widehat{y}]$ with $f(0)=g(0)=\widehat{v}$ such that for all $t \in[0,1]$, $\pi_{p}(f(t))=\pi_{p}(g(t))$.

Proof. (1) $\Rightarrow(2)$ Suppose that $A$ is $p$-symmetric. Then there is a $\widehat{v} \in A \cap \Phi_{p}$ such that $\overrightarrow{[\widehat{v}, \widehat{x}]}$ and $\overrightarrow{[\widehat{v}, \widehat{y}]}$ have the same $p$-shape $w$. Then $S_{p}(A)=w^{-1} w$.

$(2) \Rightarrow(3)$ Let $w$ be as in (2). Then $S_{p}(A)=w^{-1} w=\left(w^{-1} w\right)^{-1}=S_{p}\left(A^{\prime}\right)$.

$(3) \Rightarrow(4)$ If $A \cap \Psi_{p}$ is enumerated as $\left\{\widehat{x}_{0}, \widehat{x}_{1}, \ldots, \widehat{x}_{n}\right\}$ (in order along $A$ ), then $A^{\prime} \cap \Psi_{p}$ is enumerated in the order $\left\{\widehat{x}_{n}, \ldots, \widehat{x}_{1}, \widehat{x}_{0}\right\}$ along $A^{\prime}$, so since $A$ and $A^{\prime}$ have the same shape, $\pi_{p}\left(\widehat{x}_{i}\right)=\pi_{p}\left(\widehat{x}_{n-i}\right)$ by Proposition 4.7. If $n$ were odd, then we would have $\pi_{p}\left(\widehat{x}_{i}\right)=\pi_{p}\left(\widehat{x}_{i+1}\right)$, where $i$ is the integer part of $n / 2$, contradicting that $\pi_{p}\left(\widehat{x}_{i}\right)$ and $\pi_{p}\left(\widehat{x}_{i+1}\right)$ are the endpoints of a $P$-basic interval. Thus, $n$ must be even. 
$(4) \Rightarrow(5)$ Follows immediately from Proposition $4.7(3) \Rightarrow(2)$.

$(5) \Rightarrow(6)$ If $h$ is as in (5), let $\widehat{v}$ be the fixed point of $h$, let $f:[0,1] \rightarrow[\widehat{v}, \widehat{x}]$ be a homeomorphism, and let $g=h \circ f$.

$(6) \Rightarrow(1)$ Trivial.

Proposition 4.9. Every $p$-arc $A$ is also a $p+1$-arc, and $W_{p+1}(A)=$ $W_{p}(\widehat{\sigma}(A))=B^{T} W_{p}(A)$.

Proof. Since $\Phi_{p} \subseteq \Phi_{p+1}$, every $p$-arc is also a $p+1$-arc. Suppose that $\pi_{p}(A)$ is the $P$-basic interval $I_{n}$. Then $W_{p}(A)$ has a 1 in the $n$th coordinate and a 0 in the other coordinates, and therefore $B^{T} W_{p}(A)$ is the $n$th column of $B^{T}$, i.e., $B^{T} W_{p}(A)$ has a 1 in the $i$ th coordinate if $I_{i} \subseteq \sigma\left(I_{n}\right)$ and a 0 otherwise. Thus, since $\pi_{p+1}(A)=\pi_{p}(\widehat{\sigma}(A))=\sigma\left(\pi_{p}(A)\right)=\sigma\left(I_{n}\right)$, since $\pi_{p+1}$ is one-to-one on $A$, and since $\pi_{p}$ is one-to-one on $\widehat{\sigma}(A)$, we have $W_{p+1}(A)=$ $W_{p}(\widehat{\sigma}(A))=B^{T} W_{p}(A)$. Since the result holds for arcs mapping to a basic interval, the additive nature of $W_{p}$ guarantees that it holds for other arcs as well.

Lemma 4.10. If $A$ is a p-arc, then there is an integer $n$ such that for all $m \geq n, W_{p}\left(\sigma^{m+1}(A)\right)>W_{p}\left(\sigma^{m}(A)\right)$.

Proof. Let $\bar{w}=W_{p}(A)$. Since $\sigma \mid T$ is locally eventually onto, there is a positive integer $j$ so that $B^{j}$ has all entries positive, so by the PerronFrobenius Theorem, the spectral radius $\lambda>1$ of $B^{T}$ is a geometrically simple eigenvalue of $B^{T}$ with a corresponding eigenvector $\bar{v}$ with $\|\bar{v}\|=1$ having all entries positive, and all other eigenvalues of $B^{T}$ have absolute value less than $\lambda$. Since $\left(B^{T}\right)^{n} \bar{w} /\left\|\left(B^{T}\right)^{n} \bar{w}\right\|$ converges to $\bar{v}$, and $B^{T} \bar{v}=\lambda \bar{v}>\bar{v}$, there is an $n$ such that $m \geq n$ implies that

$$
B^{T} \frac{\left(B^{T}\right)^{m} \bar{w}}{\left\|\left(B^{T}\right)^{m} \bar{w}\right\|}>\frac{\left(B^{T}\right)^{m} \bar{w}}{\left\|\left(B^{T}\right)^{m} \bar{w}\right\|} .
$$

By Proposition 4.9, we have $W_{p}\left(\sigma^{m+1}(A)\right)>W_{p}\left(\sigma^{m}(A)\right)$ for $m \geq n$.

Proposition 4.11. If $A$ is a $p$-arc, then $S_{p+1}(A)=S_{p}(\widehat{\sigma}(A))=B^{*}\left(S_{p}(A)\right)$.

Proof. Similar to the proof of Proposition 4.9. It is easily seen to hold if $\pi_{p}(A)$ is a $P$-basic interval, from which the full result routinely follows.

This proposition is especially useful when the endpoint of $A$ is a periodic element of $\widehat{T}$. For example, if $\widehat{0}$ is an endpoint of $A$, then we know that $S_{p}(A)$ starts with one of the $P$-basic intervals containing $\overline{0}$ (or its inverse). Say $S_{p}(A)$ starts with $I_{j}$. Then $S_{p}\left(\sigma^{k}(A)\right)$ will start with $B^{* k}\left(I_{j}\right)$. But $A$ is a proper subarc of, and shares the endpoint $\widehat{0}$ with, $\sigma^{k}(A)$, so one of $S_{p}(A)$ and $B^{* k}\left(I_{j}\right)$ is an initial subword of the other. The latter of these is easily calculated for specific cases, and such calculations were a useful tool in experiments made at the beginning of this research. 
Corollary 4.12. If $A$ is p-symmetric, then $\sigma(A)$ is p-symmetric.

COROLlary 4.13. If $A$ is p-symmetric, then $A$ is also q-symmetric for all $q \geq p$.

If $A$ is $p$-symmetric, then it is not necessarily the case that $A$ is $p-1$ symmetric, and we would like to see in detail how the $p-1$-symmetry of $A$ might fail. If $A=[\widehat{x}, \widehat{y}]$ is $p$-symmetric with center $\widehat{z}$, then there are bijections $f:[0,1] \rightarrow[\widehat{z}, \widehat{x}]$ and $g:[0,1] \rightarrow[\widehat{z}, \widehat{y}]$ with $f(0)=g(0)=\widehat{z}$ such that for all $t \in[0,1], \pi_{p}(f(t))=\pi_{p}(g(t))$. Let $a \in[0,1]$ be maximal such that for all $t \in[0, a], \pi_{p-1}(f(t))=\pi_{p-1}(g(t))$. Then there are three cases:

CASE 1: $a=1$ and $\pi_{p-1}(f(1)) \in P$. Then $A$ is also $p-1$-symmetric.

CAse $2: a=1$ and $\pi_{p-1}(f(1)) \notin P$. Then $A$ is not $p-1$-symmetric, because its endpoints are not in $\Phi_{p-1}$, but $A$ can be extended (on both sides) to an $\operatorname{arc} A^{\prime}$ which is $p-1$-symmetric (and therefore also $p$-symmetric) with center $\widehat{z}$.

CASE 3: $a<1$. This is the most interesting case. Then since $\pi_{p}(\widehat{w})=$ $\sigma\left(\pi_{p-1}(\widehat{w})\right)$ for all $\widehat{w} \in \widehat{T}$, and $\tau^{0}$ is the only point of $\widehat{T}$ at which $\sigma$ is not locally one-to-one, $\pi_{p-1}(f(a))=\pi_{p-1}(g(a))=\tau^{0}$. We claim that $\pi_{p-1} \circ f$ and $\pi_{p-1} \circ g$ are both one-to-one on $[a, 1]$. To see this, pick $b \in[a, 1]$ maximal such that $\pi_{p-1} \circ f$ and $\pi_{p-1} \circ g$ are both one-to-one on $[a, b]$, and suppose that $b<1$. By symmetry, suppose that $\pi_{p-1} \circ f$ is not one-to-one on any neighborhood of $b$. Then $\pi_{p-1}(f(b)) \in O$. Since $\pi_{p-1}(f(t)) \neq \pi_{p-1}(g(t))$ for $t \in(a, b], \pi_{p-1}(g(b)) \notin O$. Thus $\pi_{p} \circ f$ is not one-to-one on a neighborhood of $b$, but $\pi_{p} \circ g$ is one-to-one on a neighborhood of $b$, contradicting that $A$ is $p$-symmetric, and establishing the claim. Since it follows that $\pi_{p-1}(f(1)) \neq$ $\pi_{p-1}(g(1))$, we see that $\pi_{p-1}(f(1))$ and $\pi_{p-1}(g(1))$ cannot both be in $O$. The following proposition isolates one important fact from what we have just shown.

Proposition 4.14. If $A=[\widehat{x}, \widehat{y}]$ is p-symmetric but not $p-1$ symmetric, then $\pi_{p-1}(\widehat{x})$ and $\pi_{p-1}(\widehat{y})$ are not both in $O$.

Proof. As noted above, the hypothesis implies that Case 2 or Case 3 held.

Proposition 4.15. If $A$ is a $p$-symmetric arc with center $\widehat{z}$, then $z \in \Psi_{p}$, and $L_{p}(z) \geq 1$.

Proof. Let $A^{\prime}$ be a subarc of $A$ with $\widehat{z}$ in its interior, and containing no point of $\Psi_{p}$ except $\widehat{z}$. Then $\pi_{p} \mid A^{\prime}$ is not one-to-one, so by Proposition 4.3 . there is a $\widehat{z}^{\prime} \in A^{\prime}$ such that $C\left(\widehat{z}^{\prime}\right) \leq p-1$, which clearly implies $z^{\prime} \in \Psi_{p}$. Clearly $\widehat{z}^{\prime}=\widehat{z}$ is the only possibility. 
Lemma 4.16. Let $A=[\widehat{x}, \widehat{y}]$ be a p-symmetric arc with center $\widehat{z}$, and suppose that $\widehat{x}, \widehat{y} \in \Psi_{p}$. Then $L_{p}(\widehat{z})>\min \left\{L_{p}(\widehat{x}), L_{p}(\widehat{y})\right\}$.

Proof. Let $q \leq p$ be least such that $A$ is $q$-symmetric. Then by Proposition 4.14, $\pi_{q-1}(\widehat{x})$ and $\pi_{q-1}(\widehat{y})$ are not both in $O$. Thus, $\min \left\{L_{q}(\widehat{x}), L_{q}(\widehat{y})\right\}$ $\leq 0$. On the other hand, $L_{q}(\widehat{z}) \geq 1$. Using the fact that $L_{p}(\widehat{u})=L_{q}(\widehat{u})+$ $(p-q)$ for $\widehat{u}=\widehat{x}, \widehat{y}, \widehat{z}$ gives the result.

One fact that was very useful in the proofs in [K1], [K2], and [BJKK] for the tent maps on the arc was that there were many symmetric arcs, one of whose endpoints was one of the endpoints of the inverse limit space. This is not the case for the more general tree maps covered here. Even in the simpler cases, there are no obvious symmetric arcs having the $\widehat{\tau}^{n}$ 's as endpoints on which to base the argument. Fortunately, for the stars (and many other trees) we have a suitable alternative by looking at arcs whose endpoints are the branching point $\widehat{0}$.

Lemma 4.17. Let $\widehat{x}=\overline{0} 1 . \overline{0}$. Then $\widehat{x}$ is a point of $\widehat{T}$. Let $A$ be the arc in $\widehat{T}_{\tau}$ between $\widehat{0}$ and $\widehat{x}$. Then for every $n, p \in \mathbb{Z}$ such that $-n \leq p, \sigma^{n}(A)$ is p-symmetric with center $\sigma^{n}(\overline{0} * . \tau)$.

Proof. Let $X$ be the smallest subtree of $T$ containing $\left\{\tau^{0}, \tau^{1}, \ldots, \tau^{k-1}\right\}$, and let $Y$ be the smallest subtree of $T$ containing $\left\{\tau^{1}, \tau^{2}, \ldots, \tau^{k}\right\}$. Then $X \subseteq Y$ and $\sigma$ maps $X$ one-to-one onto $Y$. Thus, if $x$ is any point of $Y$ that is not an endpoint of $Y$, then $0 x$ is a point of $X$ which is not an endpoint of $X$. Thus, since all points of $X$ other than $\tau^{0}$ start with a 0 , if $x$ is any point of $Y$ which is not an endpoint of $Y$, then $0^{n} x \in X$ for all positive integers $n$. Since (6) of Definition 3.11 implies that $1 \overline{0}$ is in $Y$ (and it is not an endpoint of $Y), 0^{n} 1 \overline{0} \in T$ for all $n$. Thus, $\overline{0} 1 . \overline{0}$ is a point of $\widehat{T}$.

Clearly, it is enough to show that $A$ is 0 -symmetric. Note that $\mu(\widehat{0}, \widehat{x})=$ $\overline{0} * . \tau$. Continuing with the $\mu$-process, we have $\mu(\widehat{0}, \overline{0} * . \tau)=\overline{0} \cdot 0^{k-1} * \tau$ and $\mu(\widehat{x}, \overline{0} * . \tau)=\overline{0} 1.0^{k-1} * \tau$. It is easy to see that all further applications of the $\mu$-process are symmetric with respect to $\overline{0} * . \tau$.

Definition 4.18. Let $A$ be the arc $[\widehat{0}, \overline{0} 1 . \overline{0}]$ from the previous lemma. For the remainder of this section, fix $A_{p, i}=\sigma^{-p+i}(A), p \in \mathbb{Z}, i \in \omega$. The $A_{p, i}$ 's will be called the $p$-standard arcs. Note that $A_{p, i}=A_{q, j}$ if and only if $-p+i=-q+j$.

Proposition 4.19.

(1) $A_{p, i}$ is a subset of $R_{-p+i}$.

(2) $A_{p, i+k}$ contains $A_{p, i}$, and also contains another arc $C$ disjoint from $A_{p, i}$ and of the same $p$-shape as $A_{p, i}$. Also, $C$ and $A_{p, i+k}$ share an endpoint.

(3) Let $\left\{\widehat{v}_{n}: n \in \omega\right\}$ be the centers of the p-standard arcs on $R_{i}$, enumerated in order along $R_{i}$. Then $L_{p}\left(\widehat{v}_{n+1}\right)=L_{p}\left(\widehat{v}_{n}\right)+k$. 
Proof. (1) is immediate from the definitions of $A_{p, i}$ and $R_{j}$. (3) is immediate from the fact that $\widehat{v}_{n+1}=\sigma^{k}\left(\widehat{v}_{n}\right)$. Let $u, v, w \in \mathcal{I}^{*}$ respectively indicate paths in $T$ from $\overline{0}$ to $\tau, \overline{0}$ to $\tau^{k}$, and $\overline{0}$ to $\tau^{k+1}$. Since $[\overline{0}, \tau]$ maps one-to-one onto $\left[\overline{0}, \tau^{k}\right]$ via $\sigma^{k-1}$, we have $\left(B^{*}\right)^{k-1}(u)=v$. Similarly, since $\left[\overline{0}, \tau^{0}\right]$ maps via $\sigma$ to $[\overline{0}, \tau]$ and $\left[\tau^{0}, \tau^{k}\right]$ maps via $\sigma$ to $\left[\tau, \tau^{k+1}\right]$ (which contains $\overline{0}$ by part (6) of Definition 3.11), $B^{*}(v)=u u^{-1} w$. Now, $S_{0}\left(A_{0,0}\right)=u u^{-1}$, and thus $S_{0}\left(A_{0, k}\right)=\left(B^{*}\right)^{k}\left(S_{0}\left(A_{0,0}\right)\right)=u u^{-1} w w^{-1} u u^{-1}$. Thus, (2) holds for $A_{0, k}$ and $A_{0,0}$. All other instances of (2) follow easily from this case.

Proposition 4.20. Let $\left\{\widehat{v}^{n}: n \in \omega\right\}$ be the centers of the $p$-standard arcs on $R_{i}$, enumerated in order along $R_{i}$. Let $\widehat{w} \in\left(\widehat{v}^{n}, \widehat{v}^{n+1}\right) \cap \Psi_{p}$. Then $L_{p}(\widehat{w})<L_{p}\left(\widehat{v}^{n}\right)$.

Proof. Every $\widehat{v}^{n}$ is equal to $\sigma^{i}(\overline{0} . * \tau)$ for some $i$. For example, if $\widehat{v}^{n}=$ $\overline{0} .0^{k} * \tau$, then $\widehat{v}^{n+1}=\sigma^{k}\left(\widehat{v}^{n}\right)=\overline{0}$. $* \tau$. Since $\tau$ starts with $k-10$ 's, $\widehat{v}_{i}^{n} \approx \widehat{v}_{i}^{n+1}$ for all $i \leq k$. Let $\widehat{w}$ be in the open arc from $\widehat{v}^{n}$ to $\widehat{v}^{n+1}$. Then, by Proposition 2.7. $\widehat{v}_{i}^{n} \approx \widehat{w}_{i} \approx \widehat{v}_{i}^{n+1}$ for all $i \leq k$, which implies that $\widehat{w} \approx \overline{0} .0^{k} 1 \bar{*}$. Then $\widehat{w}_{i}$ cannot be $*$ for $i \leq-1$, since that would give $\widehat{w}_{i+k}=1$, where $i+k<k$, a contradiction. Also $\widehat{w}_{i} \neq *$ for $1 \leq i \leq k-1$, since that would give $\widehat{w}_{k}=0$, also a contradiction. $\widehat{w}_{0}=*$ and $\widehat{w}_{k}=*$ are ruled out because $\widehat{w}$ is neither $\widehat{v}^{n+1}$ nor $\widehat{v}^{n}$. Thus, if $\widehat{w} \in \Psi_{p}$, then $L_{p}(\widehat{w})<k=L_{p}\left(\widehat{v}^{n}\right)$. All other cases of the proposition are the same as the example given, shifted by a certain number of coordinates.

Lemma 4.21. Let $A$ be a p-standard arc with center $\widehat{w}$. Let $C$ be another arc in $\widehat{T}$ with center $\widehat{v}$ and the same p-shape as $A$. Then $L_{p}(\widehat{v}) \geq L_{p}(\widehat{w})$.

Proof. We prove for $A=A_{p, n}$ by induction on $n$. If $A=A_{p, j}$ for $0 \leq j \leq$ $k-1$, then $L_{p}(\widehat{w})=j+1$, and $\pi_{p}(\widehat{w})=\pi_{p}(\widehat{v})=\tau^{j+1}$, which is an endpoint of $T$, so by Proposition 4.5, $L_{p}(\widehat{v}) \geq j+1$. Let $A=A_{p, n}$ for some $n \geq k$, and suppose that the lemma is true for $A_{p, n-k}$. Then $A_{p, n-k} \subseteq A$, and $A$ contains another $\operatorname{arc} A^{\prime}$ (with which it shares an endpoint) disjoint from $A_{p, n-k}$ and with the same shape as $A_{p, n-k}$. Since $C$ has the same shape as $A, C$ contains two disjoint arcs $D$ and $E$ (sharing an endpoint with each) having the same shape as $A_{p, n-k}$. Thus, if $\widehat{x}$ and $\widehat{y}$ are the centers of $D$ and $E$ respectively, then the induction hypothesis gives $L_{p}(\widehat{x}) \geq L_{p}(\widehat{z})$ and $L_{p}(\widehat{y}) \geq L_{p}(\widehat{z})$, where $\widehat{z}$ is the center of $A_{p, n-k}$. Since $\widehat{v}$ is the center of the $p$-symmetric $\operatorname{arc}[\widehat{x}, \widehat{y}], L_{p}(\widehat{v})>\min \left\{L_{p}(\widehat{x}), L_{p}(\widehat{y})\right\}$, so $L_{p}(\widehat{v})>L_{p}(\widehat{z})=n-k+1$. Since $\pi_{p}(\widehat{w})=\pi_{p}(\widehat{v}), L_{p}(\widehat{w})$ and $L_{p}(\widehat{v})$ are in the same congruence class modulo $n_{0}$, so since $k<n_{0}, L_{p}(\widehat{v}) \geq L_{p}(\widehat{w})=n+1$.

THEOREM 4.22. Every $p$-symmetric arc in $C(\widehat{0})$ having $\widehat{0}$ as an endpoint is one of the p-standard arcs $A_{p, i}$.

Proof. By contradiction. Suppose that there is a $p$-symmetric $\operatorname{arc} C$ having endpoints $\widehat{0}$ and $\widehat{z}$ and center $\widehat{w}$ which is not a $p$-standard arc. Then by 
Proposition 4.20 , there is a $\widehat{v}$ between $\widehat{0}$ and $\widehat{w}$ which is the center of a $p$-standard arc $A \subseteq B$ with endpoint $\widehat{0}$, such that $L_{p}(\widehat{v})>L_{p}(\widehat{w})$. Then by $p$-symmetry of $C$, there is an $\operatorname{arc} D$ having $\widehat{z}$ as one of its endpoints and center $\widehat{x}$ which has the same shape as $A$. Thus, by Lemma 4.21, $L_{p}(\widehat{x}) \geq L_{p}(\widehat{v})$. But this contradicts that $L_{p}(\widehat{w})>\min \left\{L_{p}(\widehat{x}), L_{p}(\widehat{v})\right\}$ (Lemma 4.16p).

Definition 4.23. Define the open covers $L_{n}$ of $T$ and $\mathcal{L}_{m, n}$ of $\widehat{T}$ as follows. For each $n \in \omega$, let $P_{n}=\sigma^{-n}(P)$. For each $x \in P_{n}$, define $l_{n}^{x}$ to be the largest connected open subset of $T$ which contains $x$ but contains no element of $P_{n}$ other than $x$. Let $\ell_{m, n}^{x}=\pi_{m}^{-1}\left(l_{n}^{x}\right), m \in \mathbb{Z}$. Let $L_{n}=\left\{l_{n}^{x}: x \in P_{n}\right\}$, and let $\mathcal{L}_{m, n}=\left\{\ell_{m, n}^{x}: x \in P_{n}\right\}$.

It is easy to check that the $L_{n}$ 's and $\mathcal{L}_{m, n}$ 's are tree chainings, i.e., if $U$ and $V$ are distinct elements of $\mathcal{L}_{m, n}$ (resp. $L_{n}$ ), then there is a unique sequence $U_{0}, U_{1}, \ldots, U_{r}$ of distinct elements of $\mathcal{L}_{m, n}$ (resp. $L_{n}$ ) such that $U_{i}$ intersects $U_{j}$ if and only if $|i-j| \leq 1, U_{0}=U$, and $U_{n}=V$. Also, these covers can be made arbitrarily fine. In particular, the diameter of elements of $L_{n}$ approaches 0 as $n$ approaches $\infty$, and the diameter of elements of $\mathcal{L}_{m, n}$ approaches 0 as both $-m$ and $n+m$ approach $+\infty$.

For the remainder of this section, let $h: \widehat{T} \rightarrow \widehat{T}$ be a fixed homeomorphism of $\widehat{T}$. We observe that it follows from (5) of Definition 3.11 that $h(\widehat{0})=\widehat{0}$ and that $h$ permutes the $\widehat{\tau}^{j}$ 's in some manner, $0 \leq j \leq n_{0}-1$. Let $d \in \omega$ be large enough that if $x$ and $y$ are distinct elements of $P, l_{d}^{x} \cap l_{d}^{y}=\emptyset$. Let $p, m$ be such that $\mathcal{L}_{p, m}$ is a refinement of $h\left(\mathcal{L}_{0, d}\right)$ (equivalently, $h^{-1}\left(\mathcal{L}_{p, m}\right)$ is a refinement of $\left.\mathcal{L}_{0, d}\right)$. Let $q, r$ be such that $h\left(\mathcal{L}_{q, r}\right)$ is a refinement of $\mathcal{L}_{p, m}$. Note that this necessarily means that $\mathcal{L}_{q, r}$ is a refinement of $\mathcal{L}_{0, d}$, so $q \leq 0$ ( $q<0$ in all but unlikely circumstances) and $r \geq d+q$. The numbers $d, p, m, q, r$ are also now fixed for the remainder of this section.

Definition 4.24. We define a function $\widetilde{h}: \Phi_{q} \rightarrow \Phi_{p}$ as follows. If $\widehat{x} \in \Phi_{q}$, then $\pi_{q}(\widehat{x}) \in P$ and there is a $\widehat{y} \in \widehat{P}$ such that $\pi_{q}(\widehat{x})=\pi_{q}(\widehat{y})=y$. Then $\widehat{x}$ and $\widehat{y}$ are both in $\ell_{q, r}^{y}$. Let $z=\pi_{p}(h(\widehat{y})) \in P($ since $h(\widehat{P})=\widehat{P})$. Then $\ell_{p, m}^{z}$ is the only element of $\mathcal{L}_{p, m}$ containing $h(\widehat{y})$, so since $h\left(\mathcal{L}_{q, r}\right)$ refines $\mathcal{L}_{p, m}$, we have $h\left(\ell_{q, r}^{y}\right) \subseteq \ell_{p, m}^{z}$. We define $\widetilde{h}(\widehat{x})$ to be the element of $\Phi_{p}$ which is in the same component of $\ell_{p, m}^{z}$ as $h(\widehat{x})$. The function $\widetilde{h}^{\prime}: \Phi_{p} \rightarrow \Phi_{0}$ is defined similarly, using the function $h^{-1}$ and the fact that $\mathcal{L}_{p, m}$ is a refinement of $h\left(\mathcal{L}_{0, d}\right)$. If $A=\overrightarrow{[\widehat{x}, \widehat{y}]}$ is an oriented $q$-arc, then $\widetilde{h}(A)$ is defined to be the oriented $p$-arc $\left[\overrightarrow{\widetilde{h}(\widehat{x}), \widetilde{h}(\widehat{y})]}\right.$ (and similarly for unoriented arcs), and $\widetilde{h}^{\prime}\left(A^{\prime}\right)$ is defined similarly for $p$-arcs $A^{\prime}$.

Proposition 4.25. For every $\widehat{x} \in \Phi_{q}, \widetilde{h}^{\prime}(\widetilde{h}(\widehat{x}))=\widehat{x}$, and for every $q$-arc $A$ (oriented or not), $\widetilde{h}^{\prime}(\widetilde{h}(A))=A$. 
Proof. Since $\widetilde{h}(\widehat{x})$ and $h(\widehat{x})$ are in the same component of the relevant $\ell_{p, m}^{z}, \widehat{x}=h^{-1}(h(\widehat{x}))$ and $h^{-1}(\widetilde{h}(\widehat{x}))$ are in the same component of the relevant $\ell_{0, d}^{y}$

Definition 4.26. Define a substitution $H^{*}: \mathcal{I} \rightarrow \mathcal{I}^{*}$ as follows. For each basic interval $I_{j} \in \mathcal{I}$, pick an oriented $q$-arc $A_{j}$ such that $S_{q}\left(A_{j}\right)=I_{j}$ and then define $H^{*}\left(I_{j}\right)=S_{p}\left(\widetilde{h}\left(A_{j}\right)\right)$. Define another substitution $J^{*}: \mathcal{I} \rightarrow \mathcal{I}^{*}$ similarly using $\widetilde{h}^{\prime}$ : if $S_{p}\left(A_{j}^{\prime}\right)=I_{j}$, let $J^{*}\left(I_{j}\right)=S_{0}\left(\widetilde{h}^{\prime}\left(A_{j}^{\prime}\right)\right)$.

Proposition 4.27 (cf. [K1, Proposition 65]). $H^{*}$ and $J^{*}$ are independent of the choices of the oriented arcs $A_{j}$ and $A_{j}^{\prime}$.

Proof. Suppose that $A$ and $C$ are oriented $q$-arcs such that $S_{q}(A)=$ $S_{q}(C)=I_{j}$. By Proposition 4.7, there exists an orientation preserving homeomorphism $f: A \rightarrow C$ such that for every $\widehat{x} \in A, \pi_{p}(\widehat{x})=\pi_{p}(f(\widehat{x}))$. Define $\chi: \widetilde{h}(A) \cap \Phi_{p} \rightarrow \widetilde{h}(C) \cap \Phi_{p}$ as follows. Fix $\widehat{y} \in \widetilde{h}(A) \cap \Phi_{p}$, and let $\widehat{x}=h^{-1}(\widehat{y})$ and $y=\pi_{p}(\widehat{y})$. Then $\widehat{x}$ and $f(\widehat{x})$ are both elements of some $\ell_{q, r}^{u}$ such that $h\left(\ell_{q, r}^{u}\right) \subseteq \ell_{p, m}^{y}$, so let $\chi(\widehat{y})$ be the element of $\Phi_{p}$ that is in the same component of $\ell_{p, m}^{y}$ as $h(f(\widehat{x}))$. Define $\chi^{\prime}: \widetilde{h}(C) \cap \Phi_{p} \rightarrow \widetilde{h}(A) \cap \Phi_{p}$ symmetrically. Then it is easy to check that $\chi^{\prime}=\chi^{-1}$, and thus by Proposition 4.7. $S_{p}(\widetilde{h}(A))=S_{p}(\widetilde{h}(C))$. This completes the proof for $H^{*}$. The proof for $J^{*}$ is virtually identical.

COROLlary 4.28. For any oriented q-arc $A, S_{p}(\widetilde{h}(A))=H^{*}\left(S_{q}(A)\right)$, and for any oriented $p$-arc $A^{\prime}, S_{0}\left(\widetilde{h}^{\prime}\left(A^{\prime}\right)\right)=J^{*}\left(S_{p}\left(A^{\prime}\right)\right)$

Proof. Let $A$ be an oriented $q$-arc. If $\pi_{q}(A)$ is a $P$-basic interval, then the corollary is immediate from Definition 4.26 and Proposition 4.27. If $A=$ $\overrightarrow{[\widehat{x}, \widehat{y}]}$ is such that $\pi_{q}(A)$ is not a $P$-basic interval, then there is a $\widehat{v} \in A \cap \Phi_{q}$ which is distinct from $\widehat{x}$ and $\widehat{y}$, and letting $X=\overrightarrow{[\widehat{x}, \widehat{v}]}$ and $Y=\overrightarrow{[\widehat{v}, \widehat{y}]}$, we have, by induction on the length of $S_{q}(A), S_{p}(\widetilde{h}(A))=S_{p}(\widetilde{h}(X)) S_{p}(\widetilde{h}(Y))=$ $H^{*}\left(S_{q}(X)\right) H^{*}\left(S_{q}(Y)\right)=H^{*}\left(S_{q}(X) S_{q}(Y)\right)=H^{*}\left(S_{q}(A)\right)$. The proof of the second part for an oriented $p$-arc is virtually identical.

Corollary 4.29 (cf. [K1, Proposition 65]).

(1) If $A$ and $C$ are $q$-arcs with the same q-shape, then $\widetilde{h}(A)$ and $\widetilde{h}(C)$ have the same p-shape.

(2) If $A^{\prime}$ and $C^{\prime}$ are $p$-arcs with the same p-shape, then $\widetilde{h}\left(A^{\prime}\right)$ and $\widetilde{h}\left(C^{\prime}\right)$ have the same 0-shape.

Proof. Immediate from Corollary 4.28.

COROLlary 4.30. If $A$ is a q-symmetric arc, then $\widetilde{h}(A)$ is p-symmetric. Furthermore, if $\widehat{x}$ is the center of the q-symmetric arc $A$, then $\widetilde{h}(\widehat{x})$ is the center of $\widetilde{h}(A)$. The same result applies to $\widetilde{h}^{\prime}$. 


\section{Proof. Immediate from Corollary 4.28 .}

Definition 4.31. Define the matrices $H$ and $J$ as follows. The $i, j$-entry of $H$ is the number of times that $I_{j}$ appears in $H^{*}\left(I_{i}\right)$ plus the number of times that $I_{j}^{-1}$ appears in $H^{*}\left(I_{i}\right)$. $J$ is defined similarly from $J^{*}$.

Corollary 4.32. Let $A$ be a q-arc. Then $W_{p}(\widetilde{h}(A))=H^{T} W_{q}(A)$. Let $A^{\prime}$ be a p-arc. Then $W_{0}\left(\widetilde{h}^{\prime}\left(A^{\prime}\right)\right)=J^{T} W_{p}(A)$.

THEOREM 4.33. For every q-standard arc $A, \widetilde{h}(A)$ is a p-standard arc, and for every p-standard arc $A^{\prime}, \widetilde{h}^{\prime}\left(A^{\prime}\right)$ is a 0-standard arc. More precisely, there are $t, s \in \omega$, with $t+s=-q$, such that $\widetilde{h}\left(A_{q, i}\right)=A_{p, t+i}(i \geq 0)$, and $\widetilde{h}^{\prime}\left(A_{p, j}\right)=A_{0, s+j}(j \geq t)$.

Proof. Since the $p$-standard arcs are the only $p$-symmetric arcs having $\widehat{0}$ as an endpoint, and $\widetilde{h}(\widehat{0})=\widehat{0}$ (and similarly for 0-standard arcs and $\widetilde{h}^{\prime}$ ), the first sentence of the theorem is clear. It is clear that $\widetilde{h}\left(A_{q, 0}\right)=A_{p, t}$ for some $t \in \omega$. Then by Proposition 4.25, $\widetilde{h}^{\prime}\left(A_{p, t}\right)$ must be $A_{q, 0}=A_{0,-q}=A_{0, t+s}$. Since $A_{q, 0} \subseteq A_{q, k}, \widetilde{h}\left(A_{q, 0}\right) \subseteq \widetilde{h}\left(A_{q, k}\right)$, and thus $\widetilde{h}\left(A_{q, k}\right)$ must be $A_{p, t+n k}$ for some integer $n$. But since $\widetilde{h}^{\prime}\left(A_{p, t+n k}\right)=A_{q, k}=A_{0,-q+k}$ and there is no 0 -standard arc $A$ strictly between $A_{0,-q}$ and $A_{0,-q+k}$ (in the sense of proper containment), we must have $n=1$, for otherwise there would be no reasonable value for $\widetilde{h}^{\prime}\left(A_{p, n+k}\right)$. Thus, arguing similarly by induction, $\widetilde{h}\left(A_{q, n k}\right)=A_{p, t+n k}$ for all $n \in \omega$, and $\widetilde{h}^{\prime}\left(A_{p, t+n k}\right)=A_{q, n k}=A_{0, t+s+n k}$. Of course, this argument can also be given for the other congruence classes modulo $k$, but that runs into the problem that it is not obvious that distinct congruence classes modulo $k$ will give the same values of $t$ and $s$.

Here, Lemma 4.10 comes to our aid by telling us that for some positive integer $M, n \geq M$ implies that $W_{q}\left(A_{q, n}\right)<W_{q}\left(A_{q, n+1}\right)$. Corollary 4.32 then gives $W_{p}\left(\widetilde{h}\left(A_{q, n}\right)\right)<W_{p}\left(\widetilde{h}\left(A_{q, n+1}\right)\right)$ for all $n \geq M$. Since we have already shown that $\widetilde{h}\left(A_{q, n k}\right)=A_{p, t+n k}$ for all $n \in \omega$, this gives $\widetilde{h}\left(A_{q, i}\right)=A_{p, t+i}$ for all $i \geq M$, which in turn guarantees distinct congruence classes modulo $k$ will indeed give the same values of $t$ and $s$ in the above argument.

Note that for $i \geq-q+p-t, \widetilde{h}\left(A_{q, i}\right)=A_{p, t+i}=A_{q, q-p+t+i}=\sigma^{q-p+t}\left(A_{q, i}\right)$, and that $\widetilde{h}^{\prime}\left(A_{p, j}\right)=A_{0, s+j}=A_{p, p+s+j}=\sigma^{p+s}\left(A_{p, j}\right)$ for sufficiently large $j$. Thus, for $q$-standard arcs, the behavior of the homeomorphism $h$ is close to that of $\sigma^{q-p+t}$, and for $p$ standard arcs, $h^{-1}$ behaves similarly to $\sigma^{p+s}$. For this reason, for the remainder of this section we fix the integer (which can be either positive or negative) $N=q-p+t=-p-s$. Our goal is to show that $h$ and $\sigma^{N}$ map composants in the same way. Using the theorem just proved, it is now easy to show that $h$ and $\sigma^{N}$ behave in the same way on the orbit of $\widehat{\tau}$. 
Corollary 4.34. For all $j, 0 \leq j \leq k-1, h\left(\widehat{\tau}^{j}\right)=\sigma^{N}\left(\widehat{\tau}^{j}\right)$.

Proof. Since the $\widehat{\tau}^{j}$ 's are the only endpoints of $\widehat{T}, h\left(\widehat{\tau}^{j}\right)=\widehat{\tau}^{i}$ for some $i$, and thus $h\left(\ell_{q, r}^{\pi_{q}\left(\widehat{\tau}^{j}\right)}\right) \subseteq \ell_{p, m}^{\pi_{p}\left(\widehat{\tau}^{i}\right)}$. If $\widehat{x}_{a}$ is the center of $A_{q, a}$, then $\pi_{q}\left(\widehat{x}_{a}\right)$ cycles through the members of $O$ as a cycles through the nonnegative integers, so there is an $a$ with $\widehat{x}_{a} \in \ell_{q, r}^{\pi_{q}\left(\widehat{\tau}^{j}\right)}$. Then $h\left(\widehat{x}_{a}\right) \in \ell_{p, m}^{\pi_{p}\left(\widehat{\tau}^{i}\right)}$, so $\widetilde{h}\left(\widehat{x}_{a}\right)$, which is the center of $\widetilde{h}\left(A_{q, a}\right)=A_{p, a+t}=A_{q, q-p+t+a}=\sigma^{N}\left(A_{q, a}\right)$ (and therefore also equal to $\left.\sigma^{N}\left(\widehat{x}_{a}\right)\right)$, is also in $\ell_{p, m}^{\pi_{p}\left(\widehat{\tau}^{i}\right)}$. Thus, $\widehat{\tau}^{i}=\sigma^{N}\left(\widehat{\tau}^{j}\right)$.

Lemma 4.35. Suppose that $u, v \in \mathbb{Z}$ are such that $u \leq v-n_{0}-1$, and that $\widehat{z} \in \Psi_{u, 0}$ is not an endpoint of $\widehat{T}$. Then there is a v-symmetric $v$-arc $A$ with center $\widehat{z}$ having two disjoint proper subarcs $C$ and $D$, which are mirror images of each other with respect to $\widehat{z}$, such that both $C$ and $D$ have the same $v$-shape as $A_{v, i}$ for some $i \geq v-u-n_{0}-1$.

Proof. Keep in mind that $u$ and $v$ will most likely both be negative when we use this lemma. Let $E$ be the $P$-basic interval of $T$ containing $\tau$ as one endpoint, and let $a$ be the other endpoint of $E$. Without loss of generality, assume that $E$ is oriented from $a$ to $\tau$. Using part (7) of Definition 3.11, there are two intervals $I, J \subseteq E$ with $I \cap J=\{b\}$ and a positive integer $m \leq n_{0}$ such that $\sigma^{m}(b)=\tau$ and $\sigma^{m} \mid I$ and $\sigma^{m} \mid J$ are both homeomorphisms onto $[\overline{0}, \tau]$. Since $\pi_{u}(\widehat{z})=\tau_{0}, \pi_{u+1}(\widehat{z})=\tau$, and since $\widehat{z}$ is not an endpoint of $\widehat{T}$, the composant of $\widehat{z}$ contains two points $\widehat{x}, \widehat{y} \in \Phi_{u+1}$ such that the arc $A=[\widehat{x}, \widehat{y}]$ contains exactly three points of $\Phi_{u+1}$, namely $\widehat{x}, \widehat{z}, \widehat{y}$. Since $\tau$ is an endpoint of $T$, clearly $\pi_{u+1}(\widehat{x})=\pi_{u+1}(\widehat{y})=a$. Thus $S_{u+1}(A)=E E^{-1}$, and $A$ is $u+1$ symmetric. Let $C=[\widehat{x}, \widehat{z}] \cap \pi_{u+1}^{-1}(I \cup J)$, and let $D=[\widehat{y}, \widehat{z}] \cap \pi_{u+1}^{-1}(I \cup J)$. Then $C$ and $D$ are mirror images of each other with respect to $\widehat{z}$, and $C$ and $D$ are $u+1+m$-symmetric with the same $u+1+m$-shape as $A_{u+1+m, 0}$. Then $v \geq u+1+m$, and $C$ and $D$ are $v$-symmetric with the same $v$-shape as $A_{v, v-u-1-m}$, where $v-u-1-m \geq v-u-n_{0}-1$.

Lemma 4.36. For all $j \geq n_{0}+1, \widetilde{h}\left(\Psi_{q-j, 0}\right) \subseteq \Psi_{p-t-j, 0}$.

Proof. Let $j \geq n_{0}+1$. Suppose that $\widehat{z} \in \Psi_{q-j, 0}$. If $\widehat{z}$ were an endpoint of $\widehat{T}$, then $\widehat{z}$ would be in the orbit of $\widehat{\tau}$, and we would be done by Corollary 4.34, so without loss of generality assume that $\widehat{z}$ is not an endpoint of $\widehat{T}$. Then the hypotheses of Lemma 4.35 are satisfied for $u=q-j, v=q$. Thus, there is a $q$-symmetric $q$-arc $A$ with center $\widehat{z}$ having two disjoint proper subarcs $C$ and $D$, which are mirror images of each other with respect to $\widehat{z}$, such that both $C$ and $D$ have the same $q$-shape as $A_{q, i}$ for some $i \geq j-n_{0}-1$. Thus, $\widetilde{h}(A)$ has center $\widehat{w}=\widetilde{h}(\widehat{z})$, and contains two disjoint proper subarcs $C^{\prime}$ and $D^{\prime}$, which are mirror images of each other with respect to $\widetilde{h}(\widehat{z})$, such that both $C^{\prime}$ and $D^{\prime}$ have the same $p$-shape as $A_{p, t+i}=\widetilde{h}\left(A_{q, i}\right)$ for 
some $i \geq j-n_{0}-1$. Let $\widehat{x}$ and $\widehat{y}$ be the centers of $C^{\prime}$ and $D^{\prime}$ respectively. Then, by Lemma 4.21, $L_{p}(\widehat{x}), L_{p}(\widehat{y}) \geq t+i+1$. Thus, by Lemma 4.16. $L_{p}(\widehat{w})>\min \left\{L_{p}(\widehat{x}), L_{p}(\widehat{y})\right\}$, so $L_{p}(\widehat{w})>t+i+1 \geq t+j-n_{0}$. Now, since $(q-j)-(p-t-j)=N, C_{p}(\widehat{w}) \equiv 0\left(\bmod n_{0}\right)$, i.e., $L_{p}(\widehat{w}) \equiv t+j\left(\bmod n_{0}\right)$. Thus $\widehat{w} \in \Psi_{p-t-j, 0}$.

LEMma 4.37. For all $j \geq n_{0}+t+1, \widetilde{h}^{\prime}\left(\Psi_{p-j, 0}\right) \subseteq \Psi_{-s-j, 0}$.

Proof. Same as the proof of Lemma 4.36.

ThEOREM 4.38. There exists an $e \in \omega$ such that $\widetilde{h}\left(\Psi_{e, 0}\right)=\sigma^{N}\left(\Psi_{e, 0}\right)$.

Proof. For example, let $e=q-n_{0}-1$. Then by Lemma 4.36 (with $\left.j=n_{0}+1\right)$, we get $\widetilde{h}\left(\Psi_{q-n_{0}-1,0}\right) \subseteq \Psi_{p-t-n_{0}-1,0}$. Then using Lemma 4.37 (with $j=t+n_{0}+1$ ), we get $\widetilde{h}^{\prime}\left(\Psi_{p-t-n_{0}-1,0}\right) \subseteq \Psi_{-s-t-n_{0}-1,0}=\Psi_{q-n_{0}-1,0}$. Thus, $\widetilde{h}\left(\Psi_{q-n_{0}-1,0}\right)=\Psi_{p-t-n_{0}-1,0}=\Psi_{q-n_{0}-N-1,0}=\sigma^{N}\left(\Psi_{q-n_{0}-1,0}\right)$.

Definition 4.39. Let $d$ be a taxicab metric on $T$ such that if $x, y \in T$ are in the same component of $T \backslash\left\{\tau^{0}\right\}$, then $d(\sigma(x), \sigma(y))=\lambda d(x, y)$. If $\widehat{x}$ and $\widehat{y}$ are in the same composant of $\widehat{T}$, define $\bar{d}(\widehat{x}, \widehat{y})$ as follows. Let $A \subseteq \widehat{T}$ be the arc with endpoints $\widehat{x}$ and $\widehat{y}$, pick $n$ such that $\pi_{n} \mid A$ is one-to-one, and let $\bar{d}(\widehat{x}, \widehat{y})=\lambda^{-n} d\left(\pi_{n}(\widehat{x}), \pi_{n}(\widehat{y})\right.$. Since $d\left(\pi_{n}(\widehat{x}), \pi_{n}(\widehat{y})=d\left(\sigma\left(\pi_{n-1}(\widehat{x})\right), \sigma\left(\pi_{n-1}(\widehat{y})\right)\right.\right.$ $=\lambda d\left(\pi_{n-1}(\widehat{x}), \pi_{n-1}(\widehat{y})\right.$ for such $n$, the definition is independent of the choice of $n$, and $\bar{d}(\widehat{\sigma}(\widehat{x}), \widehat{\sigma}(\widehat{y}))=\lambda \bar{d}(\widehat{x}, \widehat{y})$.

LEMMA 4.40. For each $n \in \omega$, there is a positive real number $M_{n}$ such that every arc in $\widehat{T}$ of $\bar{d}$-length greater than $M_{n}$ intersects $\Psi_{n, 0}$.

Proof. Since $\sigma$ maps $T$ locally eventually onto $T$, there is a positive integer $j$ such that for any two $P$-basic intervals $I$ and $J$ in the Markov graph, there is a path of length exactly $j$ from $I$ to $J$ in the Markov graph. Note that if $A$ and $C$ are two $n-j$-arcs of $\widehat{T}$ such that $A \cap \Phi_{n-j}$ and $B \cap \Phi_{n-j}$ each contain two elements, and such that $\pi_{n-j}(A)=\pi_{n-j}(B)$, then $A$ and $B$ have the same $\bar{d}$-length. Since $\pi_{n-j}(A)$ is a $P$-basic interval in such a case, there is a positive number $M_{n}$ such that if $A \subseteq \widehat{T}$ is a $p$-arc such that $A \cap \Phi_{n-j}$ has only two elements, then $A$ has $\bar{d}$-length less than $\frac{1}{2} M_{n}$. Fix such an $M_{n}$. Then any arc $A$ of $\bar{d}$-length greater than $M_{n}$ contains an arc $C$ such that $\pi_{n-j}(C)$ is a $P$-basic interval. Then $\sigma^{j}\left(\pi_{n-j}(C)\right)$ is all of $T$, and thus $\pi_{n}(A)=T$, from which it is obvious that $A$ intersects $\Psi_{n, 0}$.

COROLlary 4.41. There exists a positive integer $M$ such that for all $\widehat{x} \in C(\widehat{0}), \bar{d}\left(h(\widehat{x}), \sigma^{N}(\widehat{x})\right)<M$.

Proof. Let $e=q-n_{0}-1$. Then $\widetilde{h}\left(\Psi_{e, 0}\right)=\sigma^{N}\left(\Psi_{e, 0}\right)$, from which it follows that for each $\widehat{x} \in \Psi_{e, 0} \cap C(\widehat{0}), \widetilde{h}(\widehat{x})=\sigma^{N}(\widehat{x})$ (since both $\widetilde{h}$ and $\sigma^{N}$ map $R_{i}$ to the appropriate $R_{j}$ in an order preserving way). Now, for $\widehat{x} \in \Psi_{e, 0}, \widetilde{h}(\widehat{x})$ is sufficiently close to $h(\widehat{x})$ that $\widetilde{h}(\widehat{x})$ is the only element of $\Psi_{e, 0}$ in the arc 
from $\widetilde{h}(\widehat{x})$ to $h(\widehat{x})$. Thus, because of the order-preserving nature of both $h$ and $\sigma^{N}$ on the $R_{i}$ 's, there cannot be three elements of $\Psi_{e, 0}$ between $h(\widehat{y})$ and $\sigma^{N}(\widehat{y})$ for any $\widehat{y} \in C(\widehat{0})$. Thus $M=3 M_{e}$ suffices, where $M_{e}$ is as in Lemma 4.40 ,

TheOREM 4.42 (cf. [BJKK, Theorem 4.3]). For all $\widehat{x} \in \widehat{T}, h(\widehat{x})$ and $\sigma^{N}(\widehat{x})$ are in the same composant of $\widehat{T}$.

Proof. Since $C(\widehat{0})$ is dense in $\widehat{T}$, there are $\widehat{x}_{n} \in C(\widehat{0})$ such that $\lim _{n} \widehat{x}_{n}=\widehat{x}$. Then for all $n \in \omega$, we have $\bar{d}\left(h\left(\widehat{x}_{n}\right), \sigma^{N}\left(\widehat{x}_{n}\right)\right)<M$. For each $n \in \omega$, let $A_{n}$ be the arc from $h\left(\widehat{x}_{n}\right)$ to $\sigma^{N}\left(\widehat{x}_{n}\right)$. Then there is a subsequence $\left\langle A_{a_{n}}\right\rangle$ of the $A_{n}$ 's which converges in the Hausdorff metric to a continuum $A$. Since $A$ clearly contains both $h(\widehat{x})=\lim _{n} h\left(\widehat{x}_{n}\right)$ and $\sigma^{N}(\widehat{x})=\lim _{n} \sigma^{N}\left(\widehat{x}_{n}\right)$, we are done if we can show that $A$ is a proper subcontinuum of $\widehat{T}$.

Let $j<0$ be such that $\lambda^{j} M$ is less than one half of the diameter of $T$, so that for each $n$, the diameter of $\pi_{j}\left(A_{n}\right)$ is less than one half of the diameter of $T$. Then it follows that $\pi_{j}(A)$ is not all of $T$. Thus, $A$ is a proper subcontinuum of $\widehat{T}$.

Definition 4.43. Let $Z$ be a set and let $f: Z \rightarrow Z$. The period spectrum of $f$, denoted $P S_{f}$, is the function whose domain is the set of all positive integers, such that if $a$ is a positive integer, then $P S_{f}(a)$ is the cardinality of the set $\{Y: Y$ is a periodic orbit of $f$ of period $a\}$. We say that $P S_{f} \leq$ $P S_{g}$ if for every positive integer $a, P S_{f}(a) \leq P S_{g}(a) . P S_{f}<P S_{g}$ means $P S_{f} \leq P S_{g}$ and $P S_{f} \neq P S_{g}$.

If $f$ is a homeomorphism of $\widehat{T}$, and $\mathcal{C}$ is the set of all composants of $\widehat{T}$, we let $f^{\#}: \mathcal{C} \rightarrow \mathcal{C}$ be given by $f^{\#}(C)=f(C)$.

Corollary 4.44. $P S_{h^{\#}}=P S_{\sigma^{|N|} \mid}$.

Proof. Theorem 4.42 is equivalent to saying that $h^{\#}=\left(\widehat{\sigma}^{N}\right)^{\#}$, and it is well known that there is a natural one-to-one correspondence between periodic points of $\widehat{\sigma}$ and periodic points and $\sigma$ (via the natural projection $\pi_{0}$ ), which in turn gives a natural one-to-one correspondence between periodic points of $\widehat{\sigma}^{N}$ and periodic points and $\sigma^{N}$ for $N>0$.

CASE 1: $N>0$. We need to show that for every positive integer $a$, every composant fixed by $\sigma^{a}$ has exactly one fixed point. Let $C$ be a composant fixed by $\sigma^{a}$. If $C$ has an endpoint or branching point $\widehat{x}$, then $\sigma^{a}(\widehat{x})=\widehat{x}$, and for any other $\widehat{y} \in C, \bar{d}\left(\sigma^{a}(\widehat{x}), \sigma^{a}(\widehat{y})\right)=\lambda^{a} \bar{d}(\widehat{x}, \widehat{y})$, so $\sigma^{a}(\widehat{y}) \neq \widehat{y}$, and $\widehat{x}$ is the only fixed point of $C$. Otherwise, $C$ can be written as the one-to-one continuous image of $\mathbb{R}$. Let $f: \mathbb{R} \rightarrow C$ be a continuous bijection such that for all $v, w \in \mathbb{R}, \bar{d}(f(v), f(w))=|w-v|$. Then $f^{-1} \circ \sigma^{a} \circ f: \mathbb{R} \rightarrow \mathbb{R}$ is an expansive homeomorphism of $\mathbb{R}$ which has exactly one fixed point $u$, and $f(u)$ will be the unique fixed point of $\sigma^{a}$ on $C$. 
CASE 2: $N=0$. Then $h^{\#}$ fixes all composants, and $\sigma^{0}$ fixes all points of $T$.

CASE 3: $N<0$. Then since $\widehat{\sigma}$ is a homeomorphism, $\widehat{\sigma}^{N}$ and $\widehat{\sigma}^{|N|}$ have the same periodic points and periods.

5. Periodic points in the families $\phi_{k, j, \lambda}$. In this section we want to examine the period spectra of various $\phi_{k, j, \lambda}$ as $k, j$, and $\lambda$ vary. Since Section 4 has ended, the variables ( $T, p, q$, etc.) which were fixed at some point during that section are released from service, and are now available for other uses.

One advantage of our identification of points with itineraries is that periodic points are easy to identify. They are the points with periodic itineraries, and their period is the same as the period of the itinerary. If we single out such an itinerary, then it is typically the case that some $\phi_{n, k, \lambda}$ have a point with that itinerary and some do not. If we can gain information about the sets $\left\{(k, j, \lambda): \phi_{k, j, \lambda}\right.$ has a point with itinerary $\left.\alpha\right\}$ for various periodic itineraries $\alpha$, we can learn more about how the period spectra of the $\phi_{k, j, \lambda}$ 's are related to each other.

If $\alpha$ is a periodic sequence from $\{0,1\}$, then it may or may not be the case that $\alpha \in T_{\tau}$. It is easy to decide whether or not $\alpha \in D_{\tau}: \alpha \in D_{\tau}$ if and only if $\alpha$ is not a substitution of $\tau$. This is a routine consequence of the definition of $\tau$-admissibility. It is more complicated to decide if $\alpha \in T_{\tau}$, but there is still a method for doing so. If $T_{\tau}$ is an arc, it is simply a matter of using the "parity lexicographical ordering" (see, e.g., [BrD], [K1], [BJKK]). If $T_{\tau}$ is not an arc, then the algorithm is more complicated (see, e.g. [Bal, Definition 3.18 and Theorem 3.19], [BS]).

In proving some of the following results about the functions $\phi_{k, j, \lambda}$, it will be convenient to have a more explicitly defined formula for the functions. Thus, let us identify the star $S_{k}$ with the union of the arcs $\left[0, \lambda^{i+1}\right] \times\{i\}$, $0 \leq i \leq k-1$, with the understanding that the points $(0, i), 0 \leq i \leq k-1$, are all identified as the same point. The function values are defined as follows:

$$
\begin{array}{r}
\phi_{k, j, \lambda}(x, i)=(\lambda x, i+1), \quad 0 \leq i \leq k-2, \\
\phi_{k, j, \lambda}(x, k-1)= \begin{cases}(\lambda x, 0), & 0 \leq x \leq 1, \\
(\lambda(2-x), 0), & 1 \leq x \leq 2, \\
(\lambda(x-2), j), & 2 \leq x \leq \lambda^{k} .\end{cases}
\end{array}
$$

With this definition of $\phi=\phi_{k, j, \lambda}$, we can view $\phi^{n}$ locally as being a function from an interval $I$ to an interval $J$, both contained in some $\left[0, \lambda^{i+1}\right] \times\{i\}$ (not necessarily the same one). This is especially useful in the neighborhood of a periodic point of $\phi^{n}$. We note that for fixed $k, j$, if $\lambda_{n}$ converges to $\lambda$, and $x_{n}$ in the domain of $\phi_{k, j, \lambda_{n}}$ converges to $x$, then $x$ is in the domain of $\phi_{k, j, \lambda}$ and $\phi_{k, j, \lambda_{n}}\left(x_{n}\right)$ converges to $\phi_{k, j, \lambda}(x)$. 
TheOrem 5.1. Let $\alpha$ be a periodic sequence from $\{0,1\}$. If $\phi_{k, j, \lambda_{0}}$ has a point with itinerary $\alpha$, then there is a neighborhood $U=\left(\lambda_{1}, \lambda_{2}\right)$ of $\lambda_{0}$ such that for all $\lambda \in U, \phi_{k, j, \lambda}$ has a point with itinerary $\alpha$.

Proof. Let $\phi=\phi_{k, j, \lambda_{0}}$, and let $x$ be the point having itinerary $\alpha$ for $\phi$. Let $p$ be the period of $\alpha$. Then near $x, \phi^{p}$ graphs as a line whose slope has absolute value greater than 1 , and is crossing the diagonal at $x$. Small perturbations of $\lambda$ give a line crossing the diagonal at some point near $x$, giving a periodic point with the same itinerary as $x$.

Lemma 5.2. If $k \geq 2$ and $\lambda \geq 2^{1 / k}$, then $(\lambda-1) k \lambda^{k-1}>\lambda^{k}-2$.

Proof. Fix $k \geq 2$, and let $f(\lambda)=(k-1) \lambda^{k}-k \lambda^{k-1}+2$. Then $f\left(2^{1 / k}\right)=$ $k\left(2-2^{(k-1) / k}\right)>0$. Also $f^{\prime}(\lambda)=k(k-1) \lambda^{k-1}-k(k-1) \lambda^{k-2}>0$ for $\lambda \geq 2^{1 / k}$, so $f$ is increasing for $\lambda \geq 2^{1 / k}$, and thus $f(\lambda)>0$ for $\lambda \geq 2^{1 / k}$. Therefore $k \lambda^{k}-k \lambda^{k-1}>\lambda^{k}-2$ for $\lambda \geq 2^{1 / k}$.

Lemma 5.3. If $k \geq 2$ and $\lambda \geq 2^{1 / k}$, then $(\lambda-1) k \lambda^{k-1}>1$.

Proof. Let $f(x)=2-2^{(x-1) / x}-1 / x$. Then $\lim _{x \rightarrow \infty} f(x)=0$. Since $f^{\prime}(x)=\left(-2^{(x-1) / x}(\log 2)+1\right) \frac{1}{x^{2}}<0$ for $x \geq 2, f$ is decreasing for $x \geq 2$, and thus $f(x)>0$ for $x \geq 2$. Thus, the formula $\lambda^{k-1}(\lambda-1)-1 / k>0$ holds for $k \geq 2$ and $\lambda=2^{1 / k}$, from which it is easily seen to hold for larger $\lambda$. The result follows by easy algebra.

In the next two results, we want to examine how the orbit of the turning point $(1, k-1)$ changes as $\lambda$ varies within the family $\phi_{\lambda}=\phi_{k, j, \lambda}$ for fixed $k$ and $j$. It is easily seen that $\phi_{\lambda}^{i}(1, k-1)=\left(\lambda^{i}, i-1\right)$ for $1 \leq i \leq k$, and that $\phi_{\lambda}^{k+1}(1, k-1)=\left(\lambda^{k}(\lambda-2), j\right)$. For larger values of $i, \phi_{\lambda}^{i}(1, k-1)$ depends heavily on the values of $k, j, \lambda$. If we ignore the second coordinate and let $x_{i}$ be the first coordinate of $\phi_{\lambda}^{i}(1, k-1)$, we can see that we get one of the three recursive formulas $x_{i+1}=\lambda x_{i}, x_{i+1}=\lambda\left(2-x_{i}\right)$, or $x_{i+1}=\lambda\left(x_{i}-2\right)$, where the first of these expressions is used for the first $k$ steps and the third expression is used at step $k+1$.

If $n>0$ is least such that $\phi_{\lambda_{0}}^{n}(1, k-1)=(1, k-1)$, then there is a neighborhood $\left(\lambda_{1}, \lambda_{2}\right)$ of $\lambda_{0}$ such that the same recursive formulas can be used to calculate $x_{i}$ for $\lambda \in\left(\lambda_{1}, \lambda_{2}\right)$ and $0 \leq i \leq n$. We are interested in seeing how $x_{i}$ (and in particular $x_{n}$ ) change as $\lambda$ is perturbed.

Lemma 5.4. $\left|d x_{i}\right| \geq k \lambda^{k-1}|d \lambda|$ for $\lambda \in\left(\lambda_{1}, \lambda_{2}\right)$ and $k \leq i \leq n$.

Proof. By induction on $i, k \leq i \leq n$. Since $x_{k}=\lambda^{k}, d x_{k}=k \lambda^{k-1} d \lambda$. Suppose that $\left|d x_{i}\right| \geq k \lambda^{k-1}|d \lambda|$. Then $d x_{i+1}=a_{i} d \lambda \pm \lambda d x_{i}$, where $a_{i}$ is one of $x_{i}, 2-x_{i}$, or $x_{i}-2$. Note that $a_{i} \leq \max \left\{1, \lambda^{k}-2\right\}$. Then

$$
\begin{aligned}
\left|d x_{i+1}\right| & \geq \lambda\left|d x_{i}\right|-\left|a_{i}\right||d \lambda| \geq \lambda\left(k \lambda^{k-1}\right)|d \lambda|-\max \left\{1, \lambda^{k}-2\right\}|d \lambda| \\
& =k \lambda^{k-1}|d \lambda|+\left((\lambda-1) k \lambda^{k-1}-\max \left\{1, \lambda^{k}-2\right\}\right)|d \lambda| \geq k \lambda^{k-1}|d \lambda| .
\end{aligned}
$$


Lemma 5.5. Let $X$ be an interval of reals, and suppose that $f: X \rightarrow X$ is continuous. Let $a \in X$ be a fixed point of $f$ and let $b$ be a nonzero real number such that $a+2 b \in X$. Let $A \subseteq X$ be the interval having endpoints $a$ and $a+b$, and let $B \subseteq X$ be the interval having endpoints $a+b$ and $a+2 b$. Suppose that $f$ is linear with slope $s>2$ on $A$ and linear with slope $-s$ on $B$. Then there are disjoint intervals $I_{0} \subseteq A$ and $I_{1} \subseteq B$ such that $f\left(I_{0}\right)=f\left(I_{1}\right)=A \cup B$, and for every $\alpha \in\{0,1\}^{\omega}$ there is a point $x_{\alpha} \in I_{0} \cup I_{1}$ such that $f^{n}\left(x_{\alpha}\right) \in I_{\alpha_{n}}$ for all $n \in \omega$.

Proof. The existence of the intervals $I_{0}$ and $I_{1}$ is an easy consequence of the fact that $s>2$. The rest is well known and uses the usual argument, outlined very briefly here: for finite sequences $\beta$ of 0 's and 1's, define, by induction on the length of the sequence, intervals $I_{\beta}$ such that $y \in I_{\beta}$ implies that $f^{n}(y) \in I_{\beta_{n}}$ for all $n$ in the domain of $\beta$. Then given $\alpha$, let $x_{\alpha}$ be the unique element of $\bigcap_{n \in \omega} I_{\alpha \mid n}$.

TheOrem 5.6. Suppose that $k, j, \lambda_{0}$ are such that $\phi_{k, j, \lambda_{0}}$ has a point with periodic itinerary $\tau=\overline{\theta *}$, where $\theta$ is a sequence of 0 's and 1 's of length $n-1$. Let $\alpha$ be the result of substituting all occurrences of $*$ in $\tau$ with 0 , and let $\beta$ be the result of substituting all occurrences of $*$ in $\tau$ with 1 . Then there is a neighborhood $\left(\lambda_{1}, \lambda_{2}\right)$ of $\lambda_{0}$ such that if $\lambda \in\left(\lambda_{1}, \lambda_{0}\right)$, then $\phi_{k, j, \lambda}$ has no point with any of the itineraries $\tau, \alpha, \beta$, or any other substitution of $\tau$, and if $\lambda \in\left(\lambda_{0}, \lambda_{2}\right)$, then $\phi_{k, j, \lambda}$ has points of period $n$ with the itineraries $\alpha$ and $\beta$ (but no point with itinerary $\tau$ ), as well as points of all itineraries of 0 's and 1 's which are substitutions of $\tau$.

Proof. Any point with an itinerary containing the symbol $*$ must have the turning point $(1, k-1)$ in its orbit, so $(1, k-1)$ has itinerary $* \tau$ in the function $\phi_{k, j, \lambda_{0}}$. Note that if $t<1$ is close to 1 , then the itinerary of $(t, k-1)$ starts with $0 \theta$, while if $t>1$ is close to 1 , the itinerary of $(t, k-1)$ starts with $1 \theta$. Let $\left(\lambda_{1}, \lambda_{2}\right)$ be a neighborhood of $\lambda_{0}$ and let $(b, c)$ be a neighborhood of 1 such that if $\lambda \in\left(\lambda_{1}, \lambda_{2}\right)$, then the itinerary of $(t, k-1)$ for $\phi_{k, j, \lambda}$ starts with $0 \theta$ for $t \in(b, 1)$, and with $1 \theta$ for $t \in(1, c)$. Let $x_{0}=1, x_{1}=\lambda, x_{2}, \cdots, x_{n}$ be functions of $\lambda$ as above. Let $p$ be the number of times the recursive formula $x_{i+1}=\lambda\left(2-x_{i}\right)$ is used to get $x_{i+1}, k+1 \leq i \leq n-1$. Since $x_{i}=\lambda^{i}$ for $1 \leq i \leq k, d x_{i}=i \lambda^{i-1} d \lambda$ for those $i$, and thus the sign of $d x_{i}$ is the same as the sign of $d \lambda$. If $i \geq k$, then $d x_{i+1}=a_{i} d \lambda \pm \lambda d x_{i}$, where $a_{i}$ is one of $x_{i}, 2-x_{i}$, or $x_{i}-2$. Thus, by Lemma 5.4, the parity of the sign of $d x_{i+1}$ depends only on the parity of the $\pm \lambda d x_{i}$ term. Thus, $d x_{i+1}$ has the same sign as $d x_{i}$ if $x_{i+1}$ is either $\lambda x_{i}$ or $\lambda\left(x_{i}-2\right)$, and $d x_{i+1}$ has the opposite sign from $d x_{i}$ if $x_{i+1}$ is $\lambda\left(2-x_{i}\right)$.

CASE 1: $p$ is even. Then $d x_{n}$ has the same sign as $d \lambda$, so $x_{n}<1$ if $\lambda<\lambda_{0}$ and $x_{n}>1$ if $\lambda>\lambda_{0}$. In a neighborhood of $(1, k-1)$, we can 


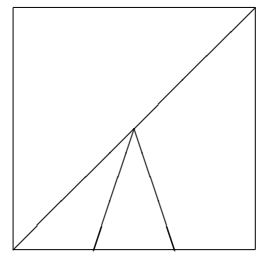

Fig. 6. $\lambda=\lambda_{0}$

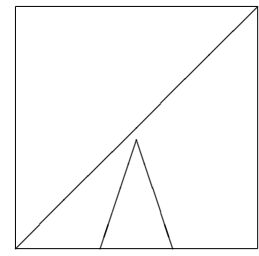

Fig. 7. $\lambda<\lambda_{0}$

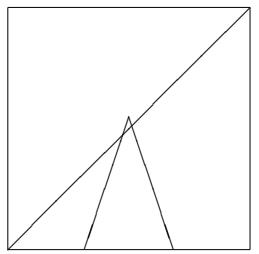

Fig. 8. $\lambda>\lambda_{0}$

view $\phi^{n}=\phi_{k, j, \lambda}^{n}$ as a map from an interval to an interval. For $\lambda=\lambda_{0}$, we have 1 (i.e., $(1, k-1)$ ) as a fixed point of $\phi^{n}$. To the left of $1, \phi^{n}$ has slope $\lambda_{0}^{n}>1$ ( $p$ changes of the sign of the slope as $\phi$ is applied $n$ times). However, to the right of $1, \phi^{n}$ has slope $-\lambda^{n}<-1$ (one change of sign on the first application of $\phi$ and then $p$ more changes of sign). Thus, for $\lambda=\lambda_{0}$, the graph of $\phi^{n}$ in the neighborhood of $(1, k-1)$ looks like Figure 6 (with the graph superimposed on the diagonal). For $\lambda \in\left(\lambda_{1}, \lambda_{0}\right), x_{n}<1$, and we get a picture like Figure 7 , with the graph in the neighborhood of $(1, k-1)$ lying below the diagonal. Here, for any point $(t, k-1)$ whose itinerary starts with $0 \theta$ or $1 \theta$, we have $\phi(t, k-1)=\left(t^{\prime}, k-1\right)$ for some $t^{\prime}<t$, and it follows that for some $i$, the itinerary of $\phi^{n i}(t, k-1)$ does not start with $0 \theta$ or $1 \theta$, so that no point has an itinerary which is a substitution of $\tau$. Finally, if $\lambda \in\left(\lambda_{0}, \lambda_{2}\right)$, then $x_{n}>1$, and we have a situation like Figure 8 . Here, there are two values near $(1, k-1)$ where the graph of $\phi^{n}$ crosses the diagonal, giving two periodic points of period $n$ in distinct orbits, one with itinerary $0 \alpha$, and the other with itinerary $1 \beta$. In fact, since $\lambda^{n}>\lambda^{k}>2$, Lemma 5.5 applies to $\phi^{n}$, giving points of all itineraries which can be formed by infinite concatenations of $0 \theta$ and $1 \theta$, thus giving points whose itineraries are any substitutions of $\tau$.

CASE 2: $p$ is odd. The argument is virtually identical to Case 1 . In this case, Figures 6,7 , and 8 need to be rotated by 180 degrees.

Definition 5.7. Let $\lambda_{k, j}$ be the unique positive root of the polynomial $\lambda^{k}-\lambda^{j}-2$. For each periodic sequence $\alpha$ from $\{0,1, *\}$, let $S(k, j, \alpha)=\{\lambda \in$ $\left(2^{1 / k}, \lambda_{k, j}\right]: \phi_{k, j, \lambda}$ has a point with itinerary $\left.\alpha\right\}$.

THEOREM 5.8. If $\alpha$ is a periodic sequence of 0 's and 1 's, then $S(k, j, \alpha)$ is an interval which, if nonempty, contains $\lambda_{k, j}$.

Proof. By Theorem 5.1. $S(k, j, \alpha)$ is an open subset of $\left(2^{1 / k}, \lambda_{k, j}\right]$. Suppose that $S(k, j, \alpha)$ is nonempty, and let $\lambda$ be the supremum of some nonempty subset $\Lambda$ of $S(k, j, \alpha)$. We need to show that $\lambda \in S(k, j, \alpha)$. There is an increasing sequence $\lambda_{n}$ from $\Lambda$ converging to $\lambda$ and points $x_{n}$ converging to a point $x$ such that $x_{n}$ has itinerary $\alpha$ in the function $\phi_{k, j, \lambda_{n}}$. Aiming for a contradiction, suppose that $x$ does not have itinerary $\alpha$. This can only 
happen if some member of the orbit of $x$ is $(1, k-1)$, so that the itinerary of $x$ is some periodic sequence $\tau$ containing the symbol $*$. This in turn means that $\alpha$ is a substitution of $\tau$. By moving to elsewhere in the orbit of $x$, we may assume without loss of generality that $\tau$ is of the form $\overline{\theta *}$. But according to Theorem 5.6, itineraries which are substitutions of $\tau$ are not possible for $\lambda^{\prime}<\lambda$ close to $\lambda$. This is a contradiction. Thus, $S(k, j, \alpha)$ is an interval, and $\lambda_{k, j} \in S(k, j, \alpha)$.

Corollary 5.9. If $\tau=\overline{\theta *}$ is periodic and acceptable, then $S(k, j, \tau)$ is either a singleton or the empty set. If $S(k, j, \tau)=\left\{\lambda_{0}\right\}$, and $\alpha$ is any substitution of $\tau$, then $S(k, j, \alpha)=\left(\lambda_{0}, \lambda_{k, j}\right]$.

TheOREM 5.10. Fix $k$ and $j$. Then $\left\{\lambda \in\left(2^{1 / k}, \lambda_{k, j}\right]: \phi_{k, j, \lambda}\right.$ has a periodic kneading sequence $\}$ is dense in $\left(2^{1 / k}, \lambda_{k, j}\right]$.

Proof. It is well known that the topological entropy of $\phi_{k, j, \lambda}$ is $\log \lambda$. If $\lambda_{1}<\lambda_{2}$, then $\phi_{k, j, \lambda_{1}}$ and $\phi_{k, j, \lambda_{2}}$ have different topological entropy, and therefore are not conjugate and have different kneading sequences. If their kneading sequences differ on the $n$th coordinate, then varying $\lambda$ continuously between $\lambda_{1}$ and $\lambda_{2}$ produces a kneading sequence with a $*$ in the $n$th coordinate, i.e., a periodic kneading sequence.

Lemma 5.11. Let $k, j \in \mathbb{Z}$, with $k \geq 2,1 \leq j \leq k-1$, and $k$ and $j$ relatively prime, and let $\lambda \in\left(2^{1 / k}, \lambda_{k, j}\right]$. Let $\overline{0}$ be the central fixed point of $\phi=\phi_{k, j, \lambda}$. Then $\phi$ has a point $z \neq \overline{0}$ of period $p$ for some $p$ such that $p \equiv(k-j)(\bmod k)$. Fix the least such $p$. If $x \neq \overline{0}$ is a point of period $n$ for $\phi$, where $n$ is not a multiple of $k$, then $p \leq n$.

Proof. Let $I_{0}, I_{1}, \ldots, I_{k-1}$ be as in the definition of $\phi_{k, j, \lambda}$, and for convenience assume that $I_{i}$ is indexed by $i \in \mathbb{Z}_{k}$. Let $J$ be the subinterval of $I_{j}$ that has preimages in $I_{k-1}$, and let $K$ be the subinterval of $I_{k-1}$ extending from the branching point $\overline{0}$ to the turning point. Define points $y_{i}$ by induction on $i$. Let $y_{0}$ be the endpoint of $I_{k-1}$ other than $\overline{0}$. Suppose that $y_{i}$ has been defined.

CASE 1: $y_{i} \in J$. Then pick $y_{i+1} \in I_{k-1}$ such that $\phi\left(y_{i+1}\right)=y_{i}$, and the induction stops at this point.

CASE 2: $y_{i} \in I_{0}$. Then pick $y_{i+1} \in K$ such that $\phi\left(y_{i+1}\right)=y_{i}$.

CASE 3: Neither Case 1 nor Case 2 holds. If $y_{i} \in I_{m}$, pick the unique $y_{i+1} \in I_{m-1}$ such that $\phi\left(y_{i+1}\right)=y_{i}$.

Since $\phi$ is locally eventually onto, there is an $i$ such that $\phi^{i}(J)$ contains $K$, and $\phi^{i+k}(J)$ contains $I_{k-1}$. Thus, it is clear that the induction eventually ends with Case 1 holding. Let $y_{p}$ be the last point picked when the induction stops. Then it is easy to see that $p \equiv(k-j)(\bmod k)$. The point $y_{p}$ will either be $y_{0}$ or a point closer to $\overline{0}$ than $y_{0}$. If $y_{p}=y_{0}$, we have found our 
point of period $p$ and we are done. If $y_{p} \neq y_{0}$, then use the "dog chases rabbit" trick to move $y$ continuously toward $\phi^{p}(y)$, starting at $y=y_{p}$. This leads to a point $z$ such that $\phi^{p}(z)=z$. Because $\overline{0}$ is a fixed point and clearly $\phi^{p}(y) \neq \overline{0}$ throughout this process, we also have $\phi^{i}(y) \neq \overline{0}$ throughout this process for all $i \leq p$, and therefore $f^{i}(z)$ and $y_{p-i}$ are in the same $I_{m}$. Thus, $z$ has period $p$.

Now, let $n>1$ be such that $n$ is not a multiple of $k$, and suppose that there is a point $x$ of period $n$ for $\phi$. To avoid trivial cases, assume $p>1$. If $x \in I_{m}$, then $f^{k-1-m}(x) \in I_{k-1}$, so at least one member of the orbit of $x$ is in $I_{k-1}$. Let $y_{0}$ be the member of the orbit of $x$ in $I_{k-1}$ which is furthest from $\overline{0}$. For $i>0$ define $y_{i}$ by induction on $i$ so that $y_{i+1}$ is the preimage of $y_{i}$ in the orbit of $x$. Then since $n$ is not a multiple of $k$, there must be a least $r>0$ such that $y_{r-1} \in I_{m}$ but $y_{r} \notin I_{m-1}$. Then $y_{r} \in I_{k-1}$ and applying the "dog chases rabbit" trick as above with $y_{r}$ and $\phi^{r}$ gives a point of period $r \equiv(k-j)(\bmod k)$, with $r \leq n$.

THEOREM 5.12. For fixed $k, P S_{\phi_{k, j_{1}, \lambda_{1}}}=P S_{\phi_{k, j_{2}, \lambda_{2}}}$ if and only if $j_{1}=j_{2}$ and $\lambda_{1}=\lambda_{2}$.

Proof. To see the nontrivial $(\Rightarrow)$ direction, suppose that either $j_{1} \neq j_{2}$ or $\lambda_{1} \neq \lambda_{2}$.

CASE 1: $j_{1}=j_{2}$. By symmetry, we may assume that $\lambda_{1}<\lambda_{2}$. Let $\tau$ be the kneading sequence of $\phi_{k, j_{1}, \lambda_{1}}$. If $\tau$ is periodic, then by Theorems 5.6 and 5.8, $\phi_{k, j_{2}, \lambda_{2}}$ has two periodic points with the same period as $\tau$ whose itineraries are substitutions of $\tau$ (along with many points of higher period whose itineraries are also substitutions of $\tau$ ). If $\alpha$ is a sequence of 0's and 1's and $\phi_{k, j_{1}, \lambda_{1}}$ has a point with itinerary $\alpha$, then so does $\phi_{k, j_{2}, \lambda_{2}}$, by Theorem 5.8. Also, there are infinitely many $\lambda \in\left(\lambda_{1}, \lambda_{2}\right)$ with period kneading sequences, and $\phi_{k, j_{2}, \lambda_{2}}$ possesses periodic points whose itineraries are substitutions of those sequences, itineraries not realized by $\phi_{k, j_{1}, \lambda_{1}}$. Thus, the period spectrum of $\phi_{k, j_{1}, \lambda_{1}}$ is strictly less than the period spectrum of $\phi_{k, j_{2}, \lambda_{2}}$.

CASE 2: $j_{1} \neq j_{2}$. For each $i \in\{1,2\}$, let $p_{i}$ be least such that $p_{i} \equiv\left(k-j_{i}\right)$ $(\bmod k)$ and $\phi_{k, j_{i}, \lambda_{i}}$ has a point of period $p_{i}$ which is different from the central fixed point. Then clearly, $p_{1} \neq p_{2}$, so by symmetry assume that $p_{1}<p_{2}$. Then, by Lemma 5.11, either $\phi_{k, j_{2}, \lambda_{2}}$ has no point of period $p_{1}$, or (in the rare case $\left.p_{1}=1\right) \phi_{k, j_{2}, \lambda_{2}}$ has only one fixed point while $\phi_{k, j_{1}, \lambda_{1}}$ has two.

Note that if we know what $P S_{f}$ is, then we can also determine $P S_{f^{n}}$ for each $n$, independent of the function $f$. That is because we know, for example, without knowing what $f$ is, that each period three orbit for $f$ leads to three period 1 orbits for $f^{3}$, that each period 5 orbit for $f$ leads to a single period 
5 orbit for $f^{3}$, that each period 12 orbit leads to three period 4 orbits for $f^{3}$, and so forth. However, the reverse is not true: $P S_{f}$ can be determined from $P S_{f^{n}}$ only in occasional trivial cases. This leads to the following definition.

Definition 5.13. Let $P_{1}$ and $P_{2}$ be functions from the set of positive integers into the class of cardinals. We say that $P_{1}$ is more primitive than $P_{2}$, written $P_{1} \triangleleft P_{2}$, if there is a function $f: Z \rightarrow Z$ for some set $Z$ such that $P S_{f}=P_{1}$ and $P S_{f^{n}}=P_{2}$ for some $n>1$. Clearly, the $\triangleleft$ relation depends only on the combinatorics of the functions $P_{1}$ and $P_{2}$. If $P_{1}(n)=0$ for all $n$, then clearly $P_{1} \triangleleft P_{2}$ (similarly if $P_{1}(n)=\kappa$ for all $n$ for any infinite cardinal $\kappa$ ), but as we see from the following results, such examples are not possible in the cases that interest us.

Proposition 5.14. Let $P_{1} \triangleleft P_{2}$ be such that $P_{1}(n)$ is finite for all $n$, and $P_{1}(n)>0$ for all but finitely many $n$. Then $P_{1} \neq P_{2}$, and if $n$ is least such that $P_{1}(n) \neq P_{2}(n)$, then $P_{1}(n)<P_{2}(n)$.

Proof. Let $k>1$ and $f$ be such that $P S_{f}=P_{1}$ and $P S_{f^{k}}=P_{2}$. Then there is a prime number $p>k$ such that $P_{1}(k p)>0$. Then $P_{2}(p)=P_{1}(p)+$ $k P_{1}(k p)>P_{1}(p)$. Let $n$ be least such that $P_{1}(n) \neq P_{2}(n)$. Then it is easy to see by induction on $m<n$ that a point has period $m$ for $f$ if and only if it has period $m$ for $f^{k}$, and that every point that has period $n$ for $f$ also has period $n$ for $f^{k}$. Thus $P_{1}(n) \leq P_{2}(n)$.

Corollary 5.15. Let $P_{1}, P_{2}$ be such that $P_{1}(n)$ is finite for all $n$, and $P_{1}(n)>0$ for all but finitely many $n$. Then it is not the case that $P_{1} \triangleleft P_{2} \triangleleft P_{1}$.

THEOREM 5.16. $\phi_{k_{1}, j_{1}, \lambda_{1}}$ and $\phi_{k_{2}, j_{2}, \lambda_{2}}$ have homeomorphic inverse limit spaces if and only if $\left(k_{1}, j_{1}, \lambda_{1}\right)=\left(k_{2}, j_{2}, \lambda_{2}\right)$.

Proof. The "if" direction is trivial. For the other direction, suppose that $\phi_{k_{1}, j_{1}, \lambda_{1}}: T_{1} \rightarrow T_{1}$ and $\phi_{k_{2}, j_{2}, \lambda_{2}}: T_{2} \rightarrow T_{2}$ have homeomorphic inverse limit spaces $\widehat{T}_{1}$ and $\widehat{T}_{2}$ with shift maps $\widehat{\sigma}_{1}$ and $\widehat{\sigma}_{2}$. Then, by Theorem 3.6, the largest $n$ such that $\widehat{T}_{i}$ contains an $n$-star is $k_{i}, i=1,2$, so $k_{1}=k_{2}$. Let $Z_{i}=$ $\left\{P S_{h \#}: h\right.$ is a homeomorphism of $\left.\widehat{T}_{i}\right\}, i \in\{1,2\}$. Then by Theorem 4.42 $Z_{i}=\left\{P S_{\left(\widehat{\sigma}_{i}^{m}\right) \#}: m \in \omega\right\}$ (noting that $P S_{\left(\widehat{\sigma}_{i}^{-m}\right)^{\#}}=P S_{\left.\left(\widehat{\sigma}_{i}^{m}\right) \#\right)}$. By Corollary 5.15, there is a unique $P_{i} \in Z_{i}$ such that $P_{i} \triangleleft P$ for all $P \in S_{i}$ such that $P \neq P_{i}$ and $P(1)$ is not infinite. Then $P S_{\phi_{k_{1}, j_{1}, \lambda_{1}}}=P_{1}=P_{2}=P S_{\phi_{k_{2}, j_{2}, \lambda_{2}}}$.

6. Problems and questions. It is natural to ask what happens if we try to generalize these results to a larger class of spaces. In particular, Hubbard Tree maps on trees other than stars are natural maps to investigate, and offer a wide variety of more general tree maps for which some of the same results might hold. Expansive Hubbard Tree maps are all conjugate to $\sigma: T_{\tau} \rightarrow T_{\tau}$ for some acceptable $\tau$ (see [Bal]). In discussing possible gen- 
eralizations, we should note that a generalized Pseudo-isotopy Conjecture does not necessarily imply a generalized Ingram's Conjecture, for there is also the problem of getting results analogous to those in Section 5. Also, a generalized Pseudo-isotopy Conjecture concerns a property of individual spaces, and can therefore be formulized in a more straightforward way than a generalized Ingram's Conjecture, which is a statement about a class of spaces. Four examples of more general Hubbard Trees, for the kneading sequences $\overline{001001 *}$ and $\overline{00101 *}, \overline{0011001100110001 *}$, and $\overline{0101 *}$, are shown in Figures 9 through 12.

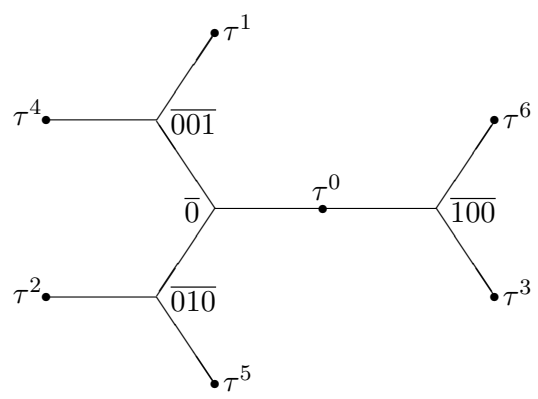

Fig. 9. Diagram of $T_{\tau}, \tau=\overline{001001 *}$

The map for $\tau=\overline{001001 *}$ (Figure 9) is an example which shows that the hypothesis that $T_{\tau}$ is a $k$-star cannot be removed from Theorem 3.9. Thus, some other proof would have to be found for the corresponding version of Lemma 4.35, or an alternative way of proving the results depending on that lemma would have to be found. There are other problems with this example which would hinder any attempt to follow the proof given here too closely. One problem is that the inverse limit space has four branching points, $\widehat{0}$, $\widehat{001}, \widehat{010}$, and $\widehat{100}$, all of valence 3 , and there does not seem to be any obvious way to guarantee that $\widehat{0}$ would be a fixed point of any homeomorphism of the inverse limit space, something that would have to be true if the Pseudoisotopy Conjecture is to be true for this map. Another problem is the lack of the $p$-standard symmetric arcs in the composant containing $\widehat{0} . T_{\tau}$ has no point with itinerary $0001 \overline{0}$, so that the inverse limit space does not contain the point $\overline{0} 1 . \overline{0}$ needed for the proof of Lemma 4.17. This last problem might be solved by altering the proof in the manner described below for the case $\tau=\overline{0101 *}$. Although it can be verified in this specific case that no other $\sigma_{\nu}: T_{\nu} \rightarrow T_{\nu}$ has a homeomorphic inverse limit by checking each of the finitely many possibilities of period 7 in Theorem 3.6, there is an infinite family of examples with larger periods whose inverse limits have the same four branching points $\widehat{0}, \widehat{001}, \widehat{010}$, and $\widehat{100}$ (routinely found from the Markov 
graph of $T_{\tau}$ ), some of which have the same periods whose inverse limits are not easily distinguished from each other.

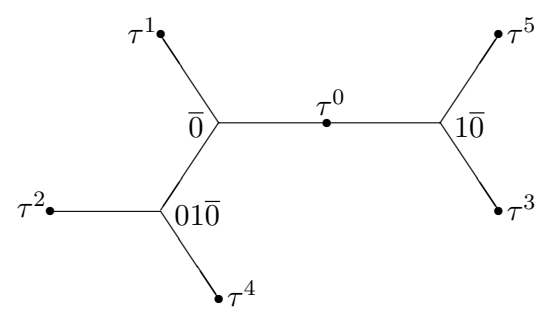

Fig. 10. Diagram of $T_{\tau}, \tau=\overline{00101 *}$

The map for $\tau=\overline{00101 *}$ (Figure 10) offers different problems. Here, $01 \overline{0}$ and $1 \overline{0}$ are only preperiodic, so that the six endpoints corresponding to the shifts of $00101 *$ and the branching point $\widehat{0}$ are the only points of the inverse limit space which are easily distinguished from the others. This leads to the problem that there is no clear definition for the set $\widehat{P}$ such that $\pi_{n}: \widehat{P} \rightarrow P$ is a bijection for all $n$. Thus, a different definition of $\Phi_{p}$ might be required, and we have not carefully investigated how that would change the arguments. This example generates an infinite family of examples whose inverse limits have only one branching point, of valence 3 , and do not obviously have different inverse limits from various examples from the families $\phi_{3, j, \lambda}$.

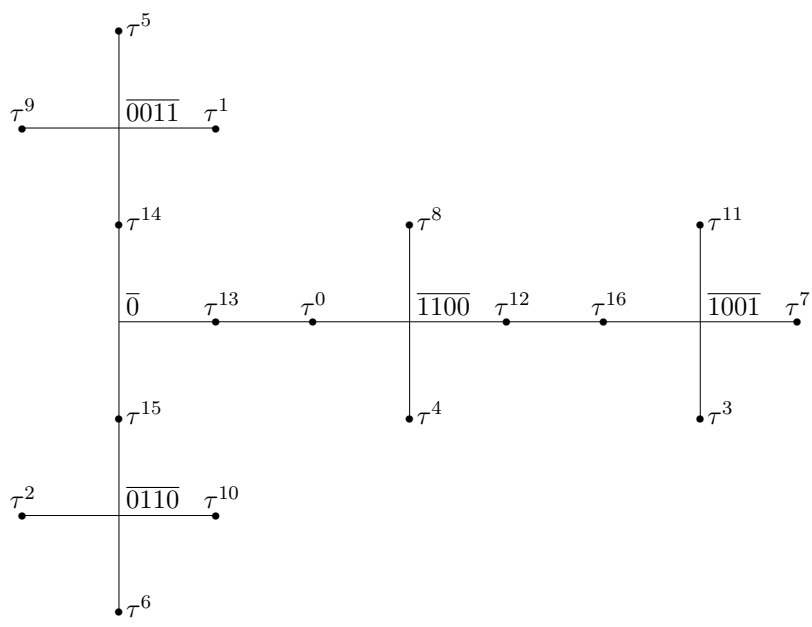

Fig. 11. Diagram of $T_{\tau}, \tau=\overline{0011001100110001 *}$

However, there are kneading sequences leading to trees which are not stars for which the arguments of Section 4 go through routinely. One example 
is $\tau=\overline{0011001100110001 *} \in \mathcal{K}$ (Figure 11). $\widehat{T}_{\tau}$ has four points of "valence" 4 , namely $\widehat{0011}, \widehat{0110}, \widehat{1100}$, and $\widehat{1001}$, but these points are permuted by any homeomorphism of $T_{\tau}$, and do not cause any problem. Many other such examples could be constructed, but closely related examples fail to go through. For example, in the simpler example with kneading sequence $\tau=\overline{001100110011} *$ (a diagram of which can be obtained from Figure 11 by erasing the points $\tau^{13}, \tau^{14}, \tau^{15}$, and $\tau^{16}$, and mapping $\tau^{12}$ to $\tau^{0}$ instead), the corresponding version of Theorem 3.9 fails. Although the Pseudo-isotopy Conjecture holds for the example of Figure 11, it is not clear whether or not this leads to an infinite family on which a generalization of Ingram's Conjecture holds.

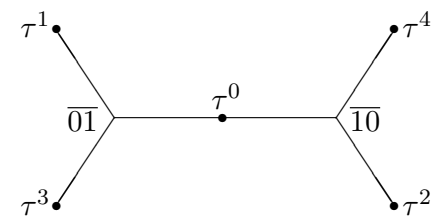

Fig. 12. Diagram of $T_{\tau}, \tau=\overline{0101 *}$

One promising modification of the argument of Section 4 can be seen in a family of Hubbard Tree maps, the simplest of which occurs with the kneading sequence $\tau=\overline{0101 *}$ (Figure 12). Here, the fixed point $\overline{0}$ has valence 2, making the corresponding point $\widehat{0}$ in the inverse limit space not very useful, but instead we have the period two points $\overline{01}$ and $\overline{10}$, corresponding to the two points $\widehat{01}$ and $\widehat{10}$ in the inverse limit space, which must be either fixed or interchanged in any homeomorphism of $T_{\tau}$. The $p$-standard arcs can then be defined in the composants containing $\widehat{01}$ and $\widehat{10}$, having one of those two points as endpoints. (The arc $A$ between $\widehat{01}$ and $\overline{01} 00 . \overline{01}$ is one $p$-standard arc.)

We close this paper by offering some questions and problems for further research. Here, the statement of an unsolved problem as a "conjecture" simply means that the statement is not now known to be false, and does not necessarily imply any strong arguments in favor of the conjecture.

Conjecture 6.1. If $\tau$ and $\nu$ are two acceptable periodic kneading sequences with range $\{0,1, *\}$ such that $\tau_{0}=\nu_{0}=0$, then $T_{\tau}$ and $T_{\nu}$ are homeomorphic if and only if $\tau=\nu$.

The reason for the hypothesis $\tau_{0}=\nu_{0}=0$ is that examples which just interchange all the 0's and 1's are clearly conjugate. Examples showing that the hypothesis that the sequences have range $\{0,1, *\}$ cannot be removed are $\sigma_{\tau}: T_{\tau} \rightarrow T_{\tau}$ for the kneading sequences $\tau=0 \overline{1}$ and $\tau=01 \overline{2} . \sigma_{0 \overline{1}}$ is conjugate to the slope 2 tent map whose inverse limit is homeomorphic 
to the Knaster Continuum. $T_{01 \overline{2}}$ is a simple triod with branching point $x_{0}$ and endpoints $x_{1}, x_{2}, x_{3}$, with $\sigma\left(x_{i}\right)=x_{i+1}$ for $i=0,1,2$ and $\sigma\left(x_{3}\right)=x_{3}$. Let $\sim$ be the smallest equivalence relation on $T_{01 \overline{2}}$ such that $s \sim t$ whenever $s \in\left[x_{0}, x_{2}\right], t \in\left[x_{0}, x_{3}\right]$ and $\sigma(s)=\sigma(t)$. Then the resulting quotient space is conjugate to $\sigma_{0 \overline{1}}$, and the corresponding semiconjugacy induces a homeomorphism between $\widehat{T}_{0 \overline{1}}$ and $\widehat{T}_{01 \overline{2}}$.

In the arguments in this paper, showing that the period spectrum of two maps was different was an important step in proving that the inverse limit spaces were different. That this is often not the case in more general settings is easily seen, for $f$ and $f^{2}$ have homeomorphic inverse limit spaces, yet $f$ and $f^{2}$ have the same period spectrum only in rare cases.

In the other direction, examples with the same period spectrum and different inverse limit spaces can be constructed using the observation that the maps $\sigma_{\tau}: T_{\tau} \rightarrow T_{\tau}$ have the same Markov graph for $\tau=01 \overline{10}$ and $\tau=00 \overline{1}$. Since there is a natural one-to-one correspondence between nonrepetitive loops in the Markov graph for $\sigma_{\tau}$ and periodic orbits for $\sigma_{\tau}$, with the period of an orbit being possibly different from the length of the loop only for the finitely many loops coming from the ray graph, a routine modification gives the following example.

EXAMPLE 6.2. Let $f_{1}=\sigma_{01 \overline{10}}: T_{1} \rightarrow T_{1}$ and let $f_{2}=\sigma_{00 \overline{1}}: T_{2} \rightarrow T_{2}$. Then $f_{1}$ and $f_{2}$ have almost identical period spectra. The Markov graph for $f_{1}$ has a nonrepetitive loop of length 4 which corresponds to an orbit of period 2, and the Markov graph for $f_{1}$ has a nonrepetitive loop of length 3 which corresponds to a fixed point. Thus, $f_{1}$ has one extra orbit of each of periods 2 and 3 that $f_{2}$ does not have, while $f_{2}$ has one extra orbit of each of periods 1 and 4 that $f_{1}$ does not have. Take a periodic point $x \in T_{1}$ such that $f_{1}(x) \neq x$ and attach five $\operatorname{arcs}[a, x],[b, x],[c, y],[d, y]$, and $[e, y]$ to $T_{1}$ at $x$, letting $f_{1}(a)=b, f_{1}(b)=c, f_{1}(c)=d, f_{1}(d)=a$, and $f_{1}(e)=e$, letting the orbits of all other new points eventually leave the new arcs. Similarly, take a periodic point $x \in T_{1}$ such that $f_{2}(x) \neq x$ and attach five $\operatorname{arcs}[a, x]$, $[b, x],[c, y],[d, y]$, and $[e, y]$ to $T_{2}$ at $x$, letting $f_{2}(a)=b, f_{2}(b)=c, f_{2}(c)=a$, $f_{2}(d)=e$, and $f_{2}(e)=d$, letting the orbits of all other new points eventually leave the new arcs. Then it is routine to check that the expanded $f_{1}$ and $f_{2}$ are Markov tree maps having identical period spectra, but different inverse limit spaces.

Question 6.3. What additional hypotheses are needed to get the result that $f$ and $g$ have the same inverse limit if and only if they have the same period spectrum?

Two continuous functions $f: X \rightarrow X$ and $g: Y \rightarrow Y$ are said to be shift-conjugate if their corresponding inverse limit shift maps $\widehat{f}$ and $\widehat{g}$ 
are conjugate. Obviously, shift-conjugate maps have homeomorphic inverse limits. Also, it is well known that $f$ and $f^{n}$ have homeomorphic inverse limits. However, we are unaware of any counterexample to the following conjecture.

ConjeCture 6.4. If $f$ and $g$ are expansive Markov tree maps, then $f$ and $g$ have homeomorphic inverse limits if and only if there are positive integers $m$ and $n$ such that $f^{m}$ and $g^{n}$ are shift-conjugate.

The "if" direction is clearly true for this conjecture. The word "expansive" certainly cannot be removed. Counterexamples would be the maps on $[0,1]$ given by $f(x)=x$ and $g(x)=x^{2}$.

It is easy to see that the core of the slope $\lambda$ tent map on the interval is conjugate to the function $\phi_{2,1, \lambda}$ of Definition 1.1. Thus, a natural generalization of the Pseudo-isotopy Conjecture is the following.

CONJECture 6.5. Let $\sigma: \widehat{S}_{k} \rightarrow \widehat{S}_{k}$ be the shift map of the inverse limit space of $\phi_{k, j, \lambda}: S_{k} \rightarrow S_{k}$, and let $h: \widehat{X} \rightarrow \widehat{X}$ be another homeomorphism of $\widehat{S}_{k}$. Then there is an integer $N$ (which need not be positive) such that for every composant $C$ of $\widehat{S}_{k}, h(C)=\sigma^{N}(C)$.

Of course, we know from the results proven above that this conjecture is true for all $\phi_{k, j, \lambda}$ having a periodic turning point. If this conjecture is true, it is likely that it would also extend to many trees which are not stars. On the other hand, the conjecture would not extend to functions $f: X \rightarrow X$ for which there exists a continuous $g: X \rightarrow X$ such that $f=g^{2}$.

Definition 6.6. Let $X$ be an indecomposable continuum, and let $\mathcal{C}(X)$ be the set of all composants of $X$. For each homeomorphism $h$ of $X$ with itself, define $h^{\#}: \mathcal{C}(X) \rightarrow \mathcal{C}(X)$ by $h^{\#}(C)=h(C)$. Let $\mathcal{G}(X)=\left\{h^{\#}: h\right.$ is a homeomorphism of $X$ \}.

Proposition 6.7. $(\mathcal{G}(X)$, o) is a group.

Proof. If $g$ and $h$ are homeomorphisms of $X$, then $g^{\#} \circ h^{\#}=(g \circ h)^{\#}$, so $(\mathcal{G}(X), \circ)$ is in fact a homomorphic image of the group of all homeomorphisms.

ThEOREM 6.8. Suppose that $\phi_{k, j, \lambda}$ has a periodic turning point, and let $\sigma: \widehat{S}_{k} \rightarrow \widehat{S}_{k}$ be the inverse limit shift map of $\phi_{k, j, \lambda}$. Then $\mathcal{G}(X)$ is a cyclic group with generator $\sigma^{\#}$.

Proof. This is an immediate corollary of Theorem 4.42 .

Conjecture 6.9. Let $f: T \rightarrow T$ be an expansive Markov tree map, and let $\sigma: \widehat{T} \rightarrow \widehat{T}$ be the corresponding inverse limit shift map. Then $\mathcal{G}(X)$ is a cyclic group. 


\section{References}

[Bal] S. Baldwin, Continuous itinerary functions and dendrite maps, Topology Appl. 154 (2007), 2889-2938.

[Ban] C. Bandt, Composants of the horseshoe, Fund. Math. 144 (1994), 231-241.

[BBS] M. Barge, H. Bruin and S. Štimac, The Ingram conjecture, preprint, 2009, 24 pp.

$[\mathrm{BaD}] \mathrm{M}$. Barge and B. Diamond, Homeomorphisms of inverse limit spaces of onedimensional maps, Fund. Math. 146 (1995), 171-187.

[BM] M. Barge and J. Martin, Endpoints of inverse limit spaces and dynamics, in: Continua with the Houston Problem Book, Lecture Notes in Pure Appl. Math. 170, Dekker, 1995, 165-182.

[BJKK] L. Block, S. Jakimovik, L. Kailhofer and J. Keesling, On the classification of inverse limits of tent maps, Fund. Math. 187 (2005), 171-192.

[BJK] L. Block, S. Jakimovik and J. Keesling, On Ingram's conjecture, Topology Proc. 30 (2006), 95-114.

[BKRS] L. Block, J. Keesling, B. Raines and S. Štimac, Homeomorphisms of unimodal inverse limit spaces with a non-recurrent critical point, Topology Appl. 156 (2009), 2417-2425.

[BrD] K. Brucks and B. Diamond, A symbolic representation of inverse limit spaces for a class of unimodal maps, in: Continua with the Houston Problem Book, Lecture Notes in Pure Appl. Math. 170, Dekker, 1995, 207-226.

[Br] H. Bruin, Inverse limit spaces of post-critically finite tent maps, Fund. Math. 165 (2000), 125-138.

[BS] H. Bruin and D. Schleicher, Symbolic dynamics of quadratic polynomials, Institut Mittag-Leffler, 2001/2, report no. 7.

[I] W. Ingram, Inverse limits on [0,1] using tent maps and certain other piecewise linear bonding maps, in: Continua with the Houston Problem Book, Lecture Notes in Pure Appl. Math. 170, Dekker, 1995, 253-258.

[K1] L. Kailhofer, A partial classification of inverse limit spaces of tent maps with periodic critical points, Topology Appl. 123 (2002), 235-265.

[K2] - A classification of inverse limit spaces of tent maps with periodic critical points, Fund. Math. 177 (2003), 95-120.

[P] W. Parry, Symbolic dynamics and transformations of the unit interval, Trans. Amer. Math. Soc. 122 (1966), 368-378.

[RS] B. Raines and S. Štimac, A classification of inverse limit spaces of tent maps with a nonrecurrent critical point, Algebr. Geom. Topology 9 (2009), 1049-1088.

[S1] S. Štimac, Structure of inverse limit spaces of tent maps with finite critical orbit, Fund. Math. 191 (2006), 125-150.

[S2] - A classification of inverse limit spaces of tent maps with finite critical orbit, Topology Appl. 154 (2007), 2265-2281.

Stewart Baldwin

Department of Mathematics and Statistics

Auburn University

Auburn, AL 36849-5310, U.S.A.

E-mail: baldwsl@auburn.edu

Received 6 April 2009;

in revised form 7 October 2009 